\title{
CHARGE AND MAGNETIC STATES FOR THE HUBBARD MODEL ON A THREE-SITE CLUSTER
}

\author{
O. P. Matveev, A. M. Shvaika \\ Institute for Condensed Matter Physics of the National Academy of Sciences of Ukraine, \\ 1 Svientsitskii St., UA-79011 Lviv, Ukraine
}

(Received May 19, 2006)

\begin{abstract}
The Hubbard model for small size clusters is investigated. Using direct diagonalization of the small cluster Hamiltonian, electronic, magnetic and spin susceptibilities are calculated and their behavior at low temperature is investigated. The temperature behavior of susceptibilities for twoand three-site clusters is investigated for both center and edge of the Brillouin zone. If at the given concentration the ground state is polarized, the susceptibilities diverge following the Curie law and the system is transformed into the magnetically ordered state at $T=0$.
\end{abstract}

Key words: Hubbard model, ground state, small clusters.

PACS number(s): 71.10.Fd, 72.10.Di, 74.25.Kc

\section{INTRODUCTION}

Compounds with transition- and rare-earth metals (oxides, sulfides and others) possess unique physical properties and attract great attention in the fields of practical applications and general-theoretical study. Regarding the magnetic properties of such compounds, they are ferromagnetic, ferrimagnetic, and antiferromagnetic. The peculiarity of some of them is a possibility of magnetic transitions which are followed by a change of the magnetic-order type under the external influence. According to the electrical properties, transition- and rareearth metal compounds may be divided into four groups: insulators, metals, compounds which possess a possibility of a metal-insulator transition (caused by external factors) with a simultaneous change of the magnetic properties and magnetic-disordered compounds in which a metal-insulator transition takes place too. But at the same time, the mechanisms of exchange interactions at the metal-insulator transition in such compounds have been studied insufficiently. This is connected with strong electron correlation. In this respect there is a need for the investigation of physics of such phenomena as ferromagnetism, antiferromagnetism, etc. by using the Hubbard model.

The Hubbard model [1-3] was originally proposed for the description of correlations in narrow-band materials on a three-dimensional lattice. It takes into account the main system characteristics, namely electron hopping and Coulomb interaction. Its two-dimensional version is often considered as the minimal model for describing the copper oxide planes in high- $T_{c}$ superconductors $[4,5]$. The one-dimensional Hubbard model has an exact solution in terms of the Bethe ansatz [6]. It displays Luttinger liquid and Mott insulator phases and has received much attention [7]. In infinite dimensions other exact solutions for the Hubbard model were obtained within Dynamical Mean Field Theory [8].

In other dimensions, for which there are no exact solutions, a great variety of approximate treatments have been proposed in order to accommodate a suitable theoretical framework. In this connection, many efforts were exerted to obtain exact ground states of few electrons for the Hubbard model on a finite size cluster (see, Ref. [9] and references therein) from which the ground state energy for the low-dimensional cases can be estimated using a concept of dimensional scaling [10].

The latest period has provided new motivations for further investigations into the systems containing a small number of particles confined in a device or unit as, for example, in the case of quantum dots, quantum well structures, mesoscopic systems, experimental entanglement, etc., for which the knowledge of the ground state does not suffice. The consideration of the excited states is a much more complicated problem. It has been studied in detail only for the two-site [11-13] and four-site [14] clusters. In Ref. [15] linear chains and rings containing two to six atoms were studied numerically and it was found that with the increase of the cluster size the thermodynamic properties of the model at half-filling approach its onedimensional limit whereas magnetic susceptibility shows a clear even-odd effect in the very low temperature region.

As there is no direct interaction between spins in the Hubbard model, it is hard to predict the behavior of a spin system. Proceeding from the fact that susceptibility of a spin system at zero temperature diverges following the Curie law [16] by means of calculating cluster susceptibilities and investigating their behavior at low temperatures one can speculate about the system order. This problem is also interesting because the phenomenon of frustrations (the problem of spins mutual accommodation) is appearing in three-site case and it is worth investigating how it influences the system ordering [17]. In particular, recently great attention was paid to the systems where the geometrical frustration exists because of an interesting phenomenon. Namely, in compounds $\mathrm{LiV}_{2} \mathrm{O}_{4}$ with the polychlorine structure a strong fermion behavior is observed [18]. Also another polychlorine compound $\mathrm{Y}(\mathrm{SC}) \mathrm{Mn}_{2}$ [19] exhibits a quantum spin-liquid behavior at low temperature. The influence of frustration is investigated for the Hubbard model on triangular lattice that is a net of tetrahedron edges in which interactions between the apexes (sites) are not the same: the 
hopping integral $\left(t^{\prime}\right)$ between the sites of base of a tetrahedron is different between the base site and the apex of a tetrahedron $(t)$ [20]. It was shown that frustration due to non-local correlations suppresses short-range antiferromagnetic fluctuations and thereby assists the formation of heavy quasi-particles near half-filling. That is why a three-site cluster is investigated in the presented work.

The aim of this work is to study the Hubbard model on a three-site cluster, namely the calculation of the energy of the many-electron states and one-electron transitions, the charge and longitudinal as well as transverse spin susceptibilities, which will contain the information about charge and magnetic states of the system.

\section{METHOD}

In this work the direct calculation of relevant Green's functions is carried out for the investigation of susceptibilities of small size clusters (two- and three-site) by means of the direct diagonalization of the Hamiltonian of the system. In such an approach the analytical expressions for magnetic and spin susceptibilities are determined in the case of the two-site cluster. For the treesite cluster numerical methods are used because the analytical expressions are too complicated. The theoretical derivation of a working formula is the same for both cases and is presented below.

By definition, Green's function built on operators $\hat{A}_{i \sigma}$, $\hat{B}_{j \sigma^{\prime}}$ is equal

$$
\mathcal{G}_{i j \sigma \sigma^{\prime}}(\tau)=-\left\langle T \hat{A}_{i \sigma}(\tau) \hat{B}_{j \sigma^{\prime}}(0)\right\rangle
$$

where $\hat{A}_{i \sigma}, \hat{B}_{j \sigma^{\prime}}$ are operators of the Bose or Fermi type, $i$ and $j$ are site indices, and $\sigma, \sigma^{\prime}=(\uparrow, \downarrow)$.

In general, all operators act in some complete basis of many particle states and can be represented by the Hubbard operators

$$
\hat{A}_{i \sigma}=\sum_{p q} A_{i \sigma}^{p q} X^{p q}, \quad \hat{B}_{j \sigma^{\prime}}=\sum_{p^{\prime} q^{\prime}} B_{j \sigma^{\prime}}^{p^{\prime} q^{\prime}} X^{p^{\prime} q^{\prime}}
$$

where $p$ and $q$ denote many particle states, then Green's function can be expressed in terms of Green's functions constructed by the Hubbard operators

$$
\mathcal{G}_{i j \sigma \sigma^{\prime}}(\tau)=-\sum_{p q p^{\prime} q^{\prime}} A_{i \sigma}^{p q} B_{j \sigma^{\prime}}^{p^{\prime} q^{\prime}}\left\langle T X^{p q}(\tau) X^{p^{\prime} q^{\prime}}(0)\right\rangle .
$$

Let us introduce a certain unitary transformation $V^{p \tilde{p}}$ which transforms the Hamiltonian of the system to a diagonal form. Then, Hubbard operators defined on the initial basis $|p\rangle$ are connected with the Hubbard operators defined on eigenstates by

$$
X^{p q}=|p\rangle\left\langle q\left|=\sum_{\tilde{p} \tilde{q}} V^{p \tilde{p}}\left(V^{q \tilde{q}}\right)^{\dagger}\right| \tilde{p}\right\rangle\langle\tilde{q}|=\sum_{\tilde{p} \tilde{q}} V^{p \tilde{p}}\left(V^{q \tilde{q}}\right)^{\dagger} X^{\tilde{p} \tilde{q}}
$$

and, respectively, Green's function equals

$$
\begin{aligned}
\mathcal{G}_{i j \sigma \sigma^{\prime}}(\tau) & =-\sum_{\substack{p q p^{\prime} q^{\prime} \\
\tilde{p} \tilde{q} \tilde{p}^{\prime} \tilde{q}^{\prime}}} A_{i \sigma}^{p q} B_{j \sigma^{\prime}}^{p^{\prime} q^{\prime}} V^{p \tilde{p}}\left(V^{q \tilde{q}}\right)^{\dagger} V^{p^{\prime} \tilde{p}^{\prime}}\left(V^{q^{\prime} \tilde{q}^{\prime}}\right)^{\dagger} \\
& \times\left\langle T X^{\tilde{p} \tilde{q}}(\tau) X^{\tilde{p}^{\prime} \tilde{q}^{\prime}}(0)\right\rangle .
\end{aligned}
$$

By definition, Heisenberg's representation for $X$ operators defined on eigenstates is reduced to [21]

$$
X^{\tilde{p} \tilde{q}}(\tau)=e^{\left(\lambda_{\tilde{p}}-\lambda_{\tilde{q}}\right) \tau} X^{\tilde{p} \tilde{q}}
$$

and introducing notations

$$
\begin{aligned}
\tilde{A}_{i \sigma}^{\tilde{p} \tilde{q}} & =\sum_{p q} V^{p \tilde{p}}\left(A_{i \sigma}^{p q} V^{q \tilde{q}}\right)^{\dagger} \\
\tilde{B}_{j \sigma^{\prime}}^{\tilde{p}^{\prime} \tilde{q}^{\prime}} & =\sum_{p^{\prime} q^{\prime}} V^{p^{\prime} \tilde{p}^{\prime}}\left(B_{j \sigma^{\prime}}^{p^{\prime} q^{\prime}} V^{q^{\prime} \tilde{q}^{\prime}}\right)^{\dagger}
\end{aligned}
$$

an expression for Green's function takes the form of

$$
\begin{aligned}
\mathcal{G}_{i j \sigma \sigma^{\prime}}(\tau) & =-\sum_{\tilde{p} \tilde{q}} \tilde{A}_{i \sigma}^{\tilde{p} \tilde{q}} \tilde{B}_{j \sigma^{\prime}}^{\tilde{q} \tilde{p}} e^{\left(\lambda_{\tilde{p}}-\lambda_{\tilde{q}}\right) \tau}\left\langle X^{\tilde{p} \tilde{p}}\right\rangle, \\
\left\langle X^{\tilde{p} \tilde{p}}\right\rangle & =\frac{e^{-\beta \lambda_{\tilde{p}}}}{\sum_{\tilde{r}} e^{-\beta \lambda_{\tilde{r}}}} .
\end{aligned}
$$

Now we pass on to an energy representation, or in other words, we carry out the Fourier transformation.

$$
\begin{gathered}
\mathcal{G}_{i j \sigma \sigma^{\prime}}\left(\omega_{n}\right)=-\int_{0}^{\beta} e^{-i \omega_{n} \tau} \sum_{\tilde{p} \tilde{q}} \tilde{A}_{i \sigma}^{\tilde{p} \tilde{q}} \tilde{B}_{j \sigma^{\prime}}^{\tilde{q} \tilde{p}} e^{\left(\lambda_{\tilde{p}}-\lambda_{\tilde{q}}\right) \tau}\left\langle X^{\tilde{p} \tilde{p}}\right\rangle d \tau \\
=-\sum_{\tilde{p} \tilde{q}} \tilde{A}_{i \sigma}^{\tilde{p} \tilde{q}} \tilde{B}_{j \sigma^{\prime}}^{\tilde{q} \tilde{p}}\left\langle X^{\tilde{p} \tilde{p}}\right\rangle \frac{1-e^{\beta\left(\lambda_{\tilde{p}}-\lambda_{\tilde{q}}-i \omega_{n}\right)}}{i \omega_{n}-\left(\lambda_{\tilde{p}}-\lambda_{\tilde{q}}\right)} .
\end{gathered}
$$

Taking into consideration that for the Bose particles $\omega_{n}=\frac{2 \pi n}{\beta}$, while for the Fermi particles $\omega_{n}=\frac{\pi(2 n+1)}{\beta}$, we obtain:

$$
\mathcal{G}_{i j \sigma \sigma^{\prime}}\left(\omega_{n}\right)=\sum_{\tilde{p} \tilde{q}} \tilde{A}_{i \sigma}^{\tilde{p} \tilde{q}} \tilde{B}_{j \sigma}^{\tilde{q} \tilde{p}} \frac{\left\langle X^{\tilde{p} \tilde{p}} \mp X^{\tilde{q} \tilde{q}}\right\rangle}{i \omega_{n}-\left(\lambda_{\tilde{p}}-\lambda_{\tilde{q}}\right)},
$$

where plus is for the Fermi particles and minus is for the Bose particles.

The given expression is final in the case of the Fermi particles, while for the Bose particles we should take into account the presence of diagonal components, namely

$$
\begin{aligned}
& \left\langle X^{\tilde{p} \tilde{p}} X^{\tilde{q} \tilde{q}}\right\rangle=\delta_{\tilde{p} \tilde{q}}\left\langle X^{\tilde{p} \tilde{p}}\right\rangle, \\
& \frac{1-e^{-i \omega_{n} \beta}}{i \omega_{n}}=\beta \delta\left(\omega_{n}\right)= \begin{cases}\frac{1}{T}, & \omega_{n}=0 \\
0, & \omega_{n} \neq 0\end{cases}
\end{aligned}
$$

Then we obtain the final expression of a working formula for Bose particles:

$$
\begin{aligned}
& \left\langle T \hat{A}_{i \sigma}(\tau) \hat{B}_{j \sigma^{\prime}}(0)\right\rangle-\left\langle\hat{A}_{i \sigma}\right\rangle\left\langle\hat{B}_{j \sigma^{\prime}}\right\rangle \\
& \stackrel{\mathrm{FT}}{\longrightarrow} \sum_{\tilde{p} \tilde{q}} \tilde{A}_{i \sigma}^{\tilde{p} \tilde{q}} \tilde{B}_{j \sigma^{\prime}}^{\tilde{q} \tilde{p}} \frac{\left\langle X^{\tilde{p} \tilde{p}}-X^{\tilde{q} \tilde{q}}\right\rangle}{i \omega_{n}-\left(\lambda_{\tilde{p}}-\lambda_{\tilde{q}}\right)}+\frac{1}{T} \delta\left(\omega_{n}\right) \\
& \times\left[\sum_{\tilde{p}} \tilde{A}_{i \sigma}^{\tilde{p} \tilde{p}} \tilde{B}_{j \sigma^{\prime}}^{\tilde{p} \tilde{p}}\left\langle X^{\tilde{p} \tilde{p}}\right\rangle-\sum_{\tilde{p}} \tilde{A}_{i \sigma}^{\tilde{p} \tilde{p}}\left\langle X^{\tilde{p} \tilde{p}}\right\rangle \sum_{\tilde{q}} \tilde{B}_{j \sigma^{\prime}}^{\tilde{q} \tilde{q}}\left\langle X^{\tilde{q} \tilde{q}}\right\rangle\right]
\end{aligned}
$$

(FT denotes the Fourier transformation). 


\section{TWO-SITE CLUSTER}

It is appropriate to consider first a simpler case of the Hubbard model on a two-site cluster. Using Green's function formalism, we calculate electronic, magnetic and spin susceptibilities and investigate their behavior in the $T \rightarrow 0$ case. It will allow us to estimate the possibilities of the ferromagnetic or antiferromagnetic ordered ground states. In the case of a two-site cluster, the problem can be solved analytically without using numerical calculations, which is practically impossible for the three-site cluster case.

\section{A. Basic equations}

Our aim is to calculate the Matsubara Green's functions $\left\langle T \hat{n}_{i}(\tau) \hat{n}_{j}(0)\right\rangle,\left\langle T \hat{m}_{i}(\tau) \hat{m}_{j}(0)\right\rangle$ and $\left\langle T \hat{S}_{i}^{+}(\tau) \hat{S}_{j}^{-}(0)\right\rangle$, where $\hat{n}_{i}=\hat{n}_{i \uparrow}+\hat{n}_{i \downarrow},\left(\hat{n}_{i \sigma}=\hat{a}_{i \sigma}^{\dagger} \hat{a}_{i \sigma}\right)$ is particle number operator, $\hat{m}_{i}=\frac{1}{2}\left(\hat{n}_{i \uparrow}-\hat{n}_{i \downarrow}\right)$ is magnetic moment operator, $\hat{S}_{i}^{+}=\hat{a}_{i \uparrow}^{\dagger} \hat{a}_{i \downarrow}, \hat{S}_{i}^{-}=\hat{a}_{i \downarrow}^{\dagger} \hat{a}_{i \uparrow}$ are spin-flip operators.

The Hamiltonian of the Hubbard model on a two-site cluster is as follows

$\mathcal{H}=\sum_{i=1,2}\left(U \hat{n}_{i \uparrow} \hat{n}_{i \downarrow}-\mu \sum_{\sigma} \hat{n}_{i \sigma}\right)+t \sum_{\sigma}\left(\hat{a}_{1 \sigma}^{\dagger} \hat{a}_{2 \sigma}+\hat{a}_{2 \sigma}^{\dagger} \hat{a}_{1 \sigma}\right)$,

where $U$ denotes the single-site Coulomb interaction, $\mu$ is a chemical potential, and $t$ is a hopping energy.

The solution of the problem in the case of a two-site cluster (density of states is calculated and energy spectra is analyzed) is shown in Ref. [12]. That is why here we will not present any details of derivation. We will apply only those results which are needed for the calculation of susceptibilities.

The initial basis of the states $|p\rangle=\left|n_{1 \uparrow}, n_{1 \downarrow}, n_{2 \uparrow}, n_{2 \downarrow}\right\rangle$ consists of sixteen states:

$$
\begin{aligned}
& |1\rangle=|0,0,0,0\rangle, \\
& |2\rangle=|0,1,0,0\rangle=\hat{a}_{1 \downarrow}^{\dagger}|1\rangle, \\
& |3\rangle=|0,0,0,1\rangle=\hat{a}_{2 \downarrow}^{\dagger}|1\rangle, \\
& |4\rangle=|1,0,0,0\rangle=\hat{a}_{1 \uparrow}^{\dagger}|1\rangle, \\
& |5\rangle=|0,0,1,0\rangle=\hat{a}_{2 \uparrow}^{\dagger}|1\rangle, \\
& |6\rangle=|0,1,0,1\rangle=\hat{a}_{2 \downarrow}^{\dagger}|2\rangle=-\hat{a}_{1 \downarrow}^{\dagger}|3\rangle, \\
& |7\rangle=|1,0,1,0\rangle=\hat{a}_{2 \uparrow}^{\dagger}|4\rangle=-\hat{a}_{1 \uparrow}^{\dagger}|5\rangle, \\
& |8\rangle=|1,1,0,0\rangle=\hat{a}_{1 \uparrow}^{\dagger}|2\rangle=-\hat{a}_{1 \downarrow}^{\dagger}|4\rangle, \\
& \left.|9\rangle=|1,0,0,1\rangle=\hat{a}_{1 \uparrow}^{\dagger}|| 3\right\rangle=-\hat{a}_{2 \downarrow}^{\dagger}|4\rangle, \\
& |10\rangle=|0,1,1,0\rangle=\hat{a}_{2 \uparrow}^{\dagger}|2\rangle=-\hat{a}_{1 \downarrow}^{\dagger}|5\rangle, \\
& |11\rangle=|0,0,1,1\rangle=\hat{a}_{2 \uparrow}^{\dagger}|3\rangle=-\hat{a}_{2 \downarrow}^{\dagger}|5\rangle, \\
& |12\rangle=|1,1,0,1\rangle=\hat{a}_{1 \uparrow}^{\dagger}|6\rangle=\hat{a}_{1 \downarrow}^{\dagger}|9\rangle=-\hat{a}_{2 \downarrow}^{\dagger}|8\rangle, \\
& |13\rangle=|0,1,1,1\rangle=\hat{a}_{2 \uparrow}^{\dagger}|6\rangle=\hat{a}_{1 \downarrow}^{\dagger}|11\rangle=-\hat{a}_{2 \downarrow}^{\dagger}|10\rangle, \\
& |14\rangle=|1,1,1,0\rangle=\hat{a}_{1 \downarrow}^{\dagger}|7\rangle=\hat{a}_{2 \uparrow}^{\dagger}|8\rangle=-\hat{a}_{1 \uparrow}^{\dagger}|10\rangle,
\end{aligned}
$$

$$
\begin{aligned}
|15\rangle & =|1,0,1,1\rangle=\hat{a}_{2 \downarrow}^{\dagger}|7\rangle=\hat{a}_{2 \uparrow}^{\dagger}|9\rangle=-\hat{a}_{1 \uparrow}^{\dagger}|11\rangle, \\
|16\rangle & =|1,1,1,1\rangle=\hat{a}_{1 \uparrow}^{\dagger}|13\rangle=-\hat{a}_{2 \uparrow}^{\dagger}|12\rangle \\
& =\hat{a}_{1 \downarrow}^{\dagger}|15\rangle=\hat{a}_{2 \downarrow}^{\dagger}|14\rangle,
\end{aligned}
$$

which, by transformation $|p\rangle=\sum_{\tilde{p}} V^{p \tilde{p}}|\tilde{p}\rangle$

$$
|\tilde{1}\rangle=|1\rangle,|\tilde{6}\rangle=|6\rangle, \quad|\tilde{7}\rangle=|7\rangle,|\widetilde{16}\rangle=|16\rangle,
$$

$$
\begin{aligned}
& \left(\begin{array}{cccc}
|2\rangle & |4\rangle & |12\rangle & |13\rangle \\
|3\rangle & |5\rangle & |14\rangle & |15\rangle
\end{array}\right) \\
& =\left\|\begin{array}{cc}
\frac{1}{\sqrt{2}} & -\frac{1}{\sqrt{2}} \\
\frac{1}{\sqrt{2}} & \frac{1}{\sqrt{2}}
\end{array}\right\|\left(\begin{array}{cccc}
|\tilde{2}\rangle & |\tilde{4}\rangle & |\widetilde{12}\rangle & |\widetilde{13}\rangle \\
|\tilde{3}\rangle & |\tilde{5}\rangle & |\widetilde{14}\rangle & |\widetilde{15}\rangle
\end{array}\right),
\end{aligned}
$$

$$
\left(\begin{array}{c}
|8\rangle \\
|9\rangle \\
|10\rangle \\
|11\rangle
\end{array}\right)=\left\|\begin{array}{rrrr}
\frac{1}{\sqrt{2}} \cos \phi & -\frac{1}{\sqrt{2}} \sin \phi & 0 & -\frac{1}{\sqrt{2}} \\
\frac{1}{\sqrt{2}} \sin \phi & \frac{1}{\sqrt{2}} \cos \phi & -\frac{1}{\sqrt{2}} & 0 \\
\frac{1}{\sqrt{2}} \sin \phi & \frac{1}{\sqrt{2}} \cos \phi & \frac{1}{\sqrt{2}} & 0 \\
\frac{1}{\sqrt{2}} \cos \phi & -\frac{1}{\sqrt{2}} \sin \phi & 0 & \frac{1}{\sqrt{2}}
\end{array}\right\|\left(\begin{array}{c}
|\tilde{8}\rangle \\
|\tilde{9}\rangle \\
|\tilde{10}\rangle \\
|\tilde{11}\rangle
\end{array}\right)
$$

where $\sin 2 \phi(t)=\frac{2 t}{\sqrt{\frac{U^{2}}{4}+4 t^{2}}}$, forms a new basis of eigenstates $|\tilde{p}\rangle$, where the Hamiltonian has a diagonal form $\mathcal{H}=\sum_{\tilde{p}} \lambda_{\tilde{p}} X^{\tilde{p} \tilde{p}}$, with the energy spectra:

$$
\begin{aligned}
\lambda_{\tilde{1}} & =0, \\
\lambda_{\tilde{2}} & =\lambda_{\tilde{4}}=-\mu+t, \\
\lambda_{\tilde{3}} & =\lambda_{\tilde{5}}=-\mu-t, \\
\lambda_{\tilde{6}} & =\lambda_{\tilde{7}}=\lambda_{\widetilde{10}}=-2 \mu, \\
\lambda_{\tilde{8}} & =U+J-2 \mu, \\
\lambda_{\tilde{9}} & =-J-2 \mu, \\
\lambda_{\widetilde{11}} & =U-2 \mu, \\
\lambda_{\widetilde{12}} & =\lambda_{\widetilde{14}}=U-3 \mu+t, \\
\lambda_{\widetilde{13}} & =\lambda_{\widetilde{15}}=U-3 \mu-t, \\
\lambda_{\widetilde{16}} & =2 U-4 \mu,
\end{aligned}
$$

and $J=\frac{4 t^{2}}{\sqrt{\frac{U^{2}}{4}+4 t^{2}}+\frac{U}{2}}$.

\section{B. Calculation of susceptibilities}

For the chain with $N=2$ periodic boundary conditions we calculate charge and magnetic susceptibilities at the center $(q=0)$ and edges $\left(q= \pm \frac{\pi}{a}\right)$ of the Brillouin zone. The charge and magnetic susceptibilities are constructed by the particle number operators which are represented by the Hubbard operators on the diagonalized basis as follows:

$$
\begin{aligned}
\hat{n}_{1 \uparrow} & =\frac{1}{2} X^{\tilde{4}, \tilde{4}}+\frac{1}{2} X^{\tilde{5}, \tilde{5}}+X^{\tilde{7}, \tilde{7}}+\frac{1}{2} X^{\tilde{8}, \tilde{8}}+\frac{1}{2} X^{\tilde{9}, \tilde{9}} \\
& +\frac{1}{2} X^{\widetilde{10}, \widetilde{10}}+\frac{1}{2} X^{\widetilde{11}, \widetilde{11}}+\frac{1}{2} X^{\widetilde{12}, \widetilde{12}}+\frac{1}{2} X^{\widetilde{13}, \widetilde{13}} \\
& +X^{\widetilde{14}, \widetilde{14}}+X^{\widetilde{15}, \widetilde{15}}+X^{\widetilde{16}, \widetilde{16}}
\end{aligned}
$$




$$
\begin{aligned}
- & \frac{1}{2}\left(X^{\tilde{4}, \tilde{5}}+X^{\tilde{5}, \tilde{4}}\right)-\frac{1}{2}\left(X^{\widetilde{12}, \widetilde{13}}+X^{\widetilde{13}, \widetilde{12}}\right) \\
& -\frac{1}{2} \sin \phi\left(X^{\tilde{8}, \widetilde{10}}+X^{\widetilde{10}, \tilde{8}}\right)-\frac{1}{2} \cos \phi\left(X^{\tilde{9}, \widetilde{10}}+X^{\widetilde{10}, \tilde{9}}\right) \\
& -\frac{1}{2} \cos \phi\left(X^{\tilde{8}, \widetilde{11}}+X^{\widetilde{11}, \tilde{8}}\right)+\frac{1}{2} \sin \phi\left(X^{\tilde{9}, \widetilde{11}}+X^{\widetilde{11}, \tilde{9}}\right), \\
\hat{n}_{1 \downarrow}= & \frac{1}{2} X^{\tilde{2}, \tilde{2}}+\frac{1}{2} X^{\tilde{3}, \tilde{3}}+X^{\tilde{6}, \tilde{6}}+\frac{1}{2} X^{\tilde{8}, \tilde{8}}+\frac{1}{2} X^{\tilde{9}, \tilde{9}}(3.18) \\
+ & \frac{1}{2} X^{\widetilde{10}, \widetilde{10}}+\frac{1}{2} X^{\widetilde{11}, \widetilde{11}}+X^{\widetilde{12}, \widetilde{12}}+X^{\widetilde{13}, \widetilde{13}} \\
+ & \frac{1}{2} X^{\widetilde{14}, \widetilde{14}}+\frac{1}{2} X^{\widetilde{15}, \widetilde{15}}+X^{\widetilde{16}, \widetilde{16}} \\
& -\frac{1}{2}\left(X^{\tilde{2}, \tilde{3}}+X^{\tilde{3}, \tilde{2}}\right)-\frac{1}{2}\left(X^{\widetilde{14}, \widetilde{15}}+X^{\widetilde{15}, \widetilde{14}}\right) \\
& +\frac{1}{2} \sin \phi\left(X^{\tilde{8}, \widetilde{10}}+X^{\widetilde{10}, \tilde{8}}\right)+\frac{1}{2} \cos \phi\left(X^{\tilde{9}, \widetilde{10}}+X^{\widetilde{10}, \tilde{9}}\right) \\
& -\frac{1}{2} \cos \phi\left(X^{\tilde{8}, \widetilde{11}}+X^{\widetilde{11}, \tilde{8}}\right)+\frac{1}{2} \sin \phi\left(X^{\tilde{9}, \widetilde{11}}+X^{\widetilde{11}, \tilde{9}}\right),
\end{aligned}
$$$$
\hat{n}_{2 \uparrow}=\frac{1}{2} X^{\tilde{4}, \tilde{4}}+\frac{1}{2} X^{\tilde{5}, \tilde{5}}+X^{\tilde{7}, \tilde{7}}+\frac{1}{2} X^{\tilde{8}, \tilde{8}}+\frac{1}{2} X^{\tilde{g}, \tilde{9}}
$$$$
+\frac{1}{2} X^{\widetilde{10}, \widetilde{10}}+\frac{1}{2} X^{\widetilde{11}, \widetilde{11}}+\frac{1}{2} X^{\widetilde{12}, \widetilde{12}}+\frac{1}{2} X^{\widetilde{13}, \widetilde{13}}
$$$$
+X^{\widetilde{14}, \widetilde{14}}+X^{\widetilde{15}, \widetilde{15}}+X^{\widetilde{16}, \widetilde{16}}
$$$$
+\frac{1}{2}\left(X^{\tilde{4}, \tilde{5}}+X^{\tilde{5}, \tilde{4}}\right)+\frac{1}{2}\left(X^{\widetilde{12}, \widetilde{13}}+X^{\widetilde{13}, \widetilde{12}}\right)
$$$$
+\frac{1}{2} \sin \phi\left(X^{\tilde{8}, \widetilde{10}}+X^{\widetilde{10}, \tilde{8}}\right)+\frac{1}{2} \cos \phi\left(X^{\tilde{9}, \widetilde{10}}+X^{\widetilde{10}, \tilde{9}}\right)
$$$$
+\frac{1}{2} \cos \phi\left(X^{\tilde{8}, \widetilde{11}}+X^{\widetilde{11}, \tilde{8}}\right)-\frac{1}{2} \sin \phi\left(X^{\tilde{9}, \widetilde{11}}+X^{\widetilde{11}, \tilde{9}}\right),
$$$$
\hat{n}_{2 \downarrow}=\frac{1}{2} X^{\tilde{2}, \tilde{2}}+\frac{1}{2} X^{\tilde{3}, \tilde{3}}+X^{\tilde{6}, \tilde{6}}+\frac{1}{2} X^{\tilde{8}, \tilde{8}}+\frac{1}{2} X^{\tilde{g}, \tilde{9}}
$$$$
+\frac{1}{2} X^{\widetilde{10}, \widetilde{10}}+\frac{1}{2} X^{\widetilde{11}, \widetilde{11}}+X^{\widetilde{12}, \widetilde{12}}+X^{\widetilde{13}, \widetilde{13}}
$$$$
+\frac{1}{2} X^{\widetilde{14}, \widetilde{14}}+\frac{1}{2} X^{\widetilde{15}, \widetilde{15}}+X^{\widetilde{16}, \widetilde{16}}
$$$$
+\frac{1}{2}\left(X^{\tilde{2}, \tilde{3}}+X^{\tilde{3}, \tilde{2}}\right)+\frac{1}{2}\left(X^{\widetilde{14}, \widetilde{15}}+X^{\widetilde{15}, \widetilde{14}}\right)
$$$$
-\frac{1}{2} \sin \phi\left(X^{\tilde{8}, \widetilde{10}}+X^{\widetilde{10}, \tilde{8}}\right)-\frac{1}{2} \cos \phi\left(X^{\tilde{9}, \widetilde{10}}+X^{\widetilde{10}, \tilde{9}}\right)
$$$$
+\frac{1}{2} \cos \phi\left(X^{\tilde{8}, \widetilde{11}}+X^{\widetilde{11}, \tilde{8}}\right)-\frac{1}{2} \sin \phi\left(X^{\tilde{9}, \widetilde{11}}+X^{\widetilde{11}, \tilde{9}}\right),
$$

Applying the method described in the previous chapter Green's functions constructed by the particle number operators are calculated

$$
\begin{aligned}
& \left\langle T \hat{n}_{1}(\tau) \hat{n}_{1}(0)\right\rangle=\left\langle T \hat{n}_{2}(\tau) \hat{n}_{2}(0)\right\rangle \\
& \stackrel{\mathrm{FT}}{=} \delta\left(\omega_{n}\right)\left[\frac{1}{4}\left(w_{\tilde{2}}+w_{\tilde{3}}+w_{\tilde{4}}+w_{\tilde{5}}\right)+w_{\tilde{6}}+w_{\tilde{7}}+w_{\tilde{8}}+w_{\tilde{9}}\right. \\
& \left.+w_{\widetilde{10}}+w_{\widetilde{11}}+\frac{9}{4}\left(w_{\widetilde{12}}+w_{\widetilde{13}}+w_{\widetilde{14}}+w_{\widetilde{15}}\right)+4 w_{\widetilde{16}}\right] \\
& +\frac{1}{4}\left(g_{\tilde{2}, \tilde{3}}+g_{\tilde{3}, \tilde{2}}+g_{\tilde{4}, \tilde{5}}+g_{\tilde{5}, \tilde{4}}+g_{\widetilde{12}, \widetilde{13}}+g_{\widetilde{13}, \widetilde{12}}+g_{\widetilde{14}, \widetilde{15}}+g_{\widetilde{15}, \widetilde{14}}\right) \\
& +\sin ^{2} \phi\left(g_{\tilde{9}, \widetilde{11}}+g_{\widetilde{11}, \tilde{9}}\right)+\cos ^{2} \phi\left(g_{\tilde{8}, \widetilde{11}}+g_{\widetilde{11}, \tilde{8}}\right)
\end{aligned}
$$

$$
\begin{aligned}
& \left\langle T \hat{n}_{1}(\tau) \hat{n}_{2}(0)\right\rangle=\left\langle T \hat{n}_{2}(\tau) \hat{n}_{1}(0)\right\rangle \\
& \stackrel{\mathrm{FT}}{=} \delta\left(\omega_{n}\right)\left[\frac{1}{4}\left(w_{\tilde{2}}+w_{\tilde{3}}+w_{\tilde{4}}+w_{\tilde{5}}\right)+w_{\tilde{6}}+w_{\tilde{7}}+w_{\tilde{8}}+w_{\tilde{9}}\right. \\
& \left.+w_{\widetilde{10}}+w_{\widetilde{11}}+\frac{9}{4}\left(w_{\widetilde{12}}+w_{\widetilde{13}}+w_{\widetilde{14}}+w_{\widetilde{15}}\right)+4 w_{\widetilde{16}}\right] \\
& +\frac{1}{4}\left(g_{\tilde{2}, \tilde{3}}+g_{\tilde{3}, \tilde{2}}+g_{\tilde{4}, \tilde{5}}+g_{\tilde{5}, \tilde{4}}+g_{\widetilde{12}, \widetilde{13}}+g_{\widetilde{13}, \widetilde{12}}+g_{\widetilde{14}, \widetilde{15}}+g_{\widetilde{15}, \widetilde{14}}\right) \\
& -\sin ^{2} \phi\left(g_{\tilde{9}, \widetilde{11}}-g_{\widetilde{11}, \tilde{9}}\right)+\cos ^{2} \phi\left(g_{\tilde{8}, \widetilde{11}}+g_{\widetilde{11}, \tilde{8}}\right)
\end{aligned}
$$

where we introduce notations $w_{\tilde{p}}=\left\langle X^{\tilde{p}, \tilde{p}}\right\rangle=\frac{e^{-\beta \lambda_{\tilde{r}}}}{\sum_{q} e^{-\beta \lambda_{q}}}$,

$$
g_{\tilde{p}, \tilde{q}}=\frac{\left\langle X^{\tilde{p}, \tilde{p}}-X^{\tilde{q}, \tilde{q}}\right\rangle}{i \omega_{n}-\left(\lambda_{\tilde{p}}-\lambda_{\tilde{q}}\right)}
$$

and take into account that $\left\langle\hat{n}_{i} \hat{n}_{j}\right\rangle=\left\langle\hat{n}_{i \uparrow} \hat{n}_{j \uparrow}\right\rangle+\left\langle\hat{n}_{i \downarrow} \hat{n}_{j \downarrow}\right\rangle+$ $\left\langle\hat{n}_{i \uparrow} \hat{n}_{j \downarrow}\right\rangle+\left\langle\hat{n}_{i \downarrow} \hat{n}_{j \uparrow}\right\rangle$

By definition the electronic susceptibility for different values of the wave vector is equal

$$
\chi_{q}=\sum_{i-j} \mathcal{G}_{i j} e^{-i q\left(R_{i}-R_{j}\right)},
$$

where $q=0, \pm \frac{\pi}{a}$ ( $a$ is a lattice constant). Then for the homogeneous $(q=0)$ electronic susceptibility we obtain only the static $\left(\omega_{n}=0\right)$ contribution:

$$
\begin{aligned}
& \chi_{c}=\sum_{i-j}\left\{\left\langle T \hat{n}_{i}(\tau) \hat{n}_{j}(0)\right\rangle-\left\langle\hat{n}_{i}\right\rangle\left\langle\hat{n}_{j}\right\rangle\right\} \\
& \stackrel{\mathrm{FT}}{=} \frac{2}{T} \delta\left(\omega_{n}\right)\left[w _ { \tilde { 1 } } \left(\frac{1}{4} w_{\tilde{2}}+\frac{1}{4} w_{\tilde{3}}+\frac{1}{4} w_{\tilde{4}}+\frac{1}{4} w_{\tilde{5}}+w_{\tilde{6}}+w_{\tilde{7}}+w_{\tilde{8}}\right.\right. \\
& \left.+w_{\tilde{9}}+w_{\widetilde{10}}+w_{\widetilde{11}}+\frac{9}{4} w_{\widetilde{12}}+\frac{9}{4} w_{\widetilde{13}}+\frac{9}{4} w_{\widetilde{14}}+\frac{9}{4} w_{\widetilde{15}}\right) \\
& +w_{\tilde{1} 6}\left(\frac{9}{4} w_{\tilde{2}}+\frac{9}{4} w_{\tilde{3}}+\frac{9}{4} w_{\tilde{4}}+\frac{9}{4} w_{\tilde{5}}+w_{\tilde{6}}+w_{\tilde{7}}+w_{\tilde{8}}\right. \\
& \left.+w_{\tilde{9}}+w_{\widetilde{10}}+w_{\widetilde{11}}+\frac{1}{4} w_{\widetilde{12}}+\frac{1}{4} w_{\widetilde{13}}+\frac{1}{4} w_{\widetilde{14}}+\frac{1}{4} w_{\widetilde{15}}\right) \\
& +\left(w_{\tilde{6}}+w_{\tilde{7}}\right)\left(\frac{1}{4} w_{\tilde{2}}+\frac{1}{4} w_{\tilde{3}}+\frac{1}{4} w_{\tilde{4}}+\frac{1}{4} w_{\tilde{5}}\right. \\
& \left.+\frac{1}{4} w_{\widetilde{12}}+\frac{1}{4} w_{\widetilde{13}}+\frac{1}{4} w_{\widetilde{14}}+\frac{1}{4} w_{\widetilde{15}}\right) \\
& +\left(w_{\tilde{4}}+w_{\tilde{5}}\right)\left(\frac{1}{4} w_{\tilde{8}}+\frac{1}{4} w_{\tilde{9}}+\frac{1}{4} w_{\widetilde{10}}+\frac{1}{4} w_{\widetilde{11}}+\frac{1}{2} w_{\widetilde{14}}+\frac{1}{2} w_{\widetilde{15}}\right) \\
& +\left(w_{\widetilde{12}}+w_{\widetilde{13}}\right)\left(\frac{1}{2} w_{\tilde{2}}+\frac{1}{2} w_{\tilde{3}}+\frac{1}{4} w_{\tilde{8}}+\frac{1}{4} w_{\tilde{9}}+\frac{1}{4} w_{\widetilde{10}}+\frac{1}{4} w_{\widetilde{11}}\right) \\
& \left.+4 w_{\tilde{1}} w_{\widetilde{16}}+\left(w_{\widetilde{12}}+w_{\widetilde{13}}\right)\left(w_{\tilde{4}}+w_{\tilde{5}}\right)+\left(w_{\widetilde{14}}+w_{\widetilde{15}}\right)\left(w_{\tilde{2}}+w_{\tilde{3}}\right)\right] .
\end{aligned}
$$

In order to investigate the susceptibility behavior at $T \rightarrow 0$ we should consider limits:

$$
\lim _{\lambda_{\tilde{p}}-\lambda_{\tilde{q}} \rightarrow 0} \frac{\left\langle X^{\tilde{p}, \tilde{p}}-X^{\tilde{q}, \tilde{q}}\right\rangle}{i \omega_{n}-\lambda_{\tilde{p}}+\lambda_{\tilde{q}}}=\left\{\begin{array}{ll}
0, & i \omega_{n} \neq 0 \\
\beta\left\langle X^{\tilde{q}, \tilde{q}}\right\rangle, & i \omega_{n}=0
\end{array},\right.
$$




$$
\lim _{\beta \rightarrow \infty} \beta\left\langle X^{\tilde{q}, \tilde{q}}\right\rangle=\left\{\begin{array}{ll}
0, & \lambda_{\tilde{q}}>\lambda_{\min } \\
\infty, & \lambda_{\tilde{q}}=\lambda_{\min }
\end{array} .\right.
$$

When the electron concentration is equal $n=1: \lambda_{\min }=$ $\lambda_{\tilde{3}}=\lambda_{\tilde{5}}=-\mu-t$; for $n=2: \lambda_{\min }=\lambda_{\tilde{9}}=-J-2 \mu$; for $n=3: \lambda_{\min }=\lambda_{\widetilde{13}}=\lambda_{\widetilde{15}}=U-3 \mu-t$, analyzing the expression and taking into account the corresponding limits one can see that at the temperatures close to zero the charge susceptibility tends to zero because the lowest energy states do not give a contribution. At the edges of the Brillouin zone $\left(q= \pm \frac{\pi}{a}\right)$, the electronic susceptibility is purely dynamical $\left(\omega_{n} \neq 0\right)$ :

$$
\begin{aligned}
& \chi_{c}=\sum_{i-j} e^{\mp i \pi(i-j)}\left\{\left\langle T \hat{n}_{i}(\tau) \hat{n}_{j}(0)\right\rangle-\left\langle\hat{n}_{i}\right\rangle\left\langle\hat{n}_{j}\right\rangle\right\} \\
& \stackrel{\mathrm{FT}}{=}\left(g_{\tilde{2}, \tilde{3}}+g_{\tilde{3}, \tilde{2}}+g_{\tilde{4}, \tilde{5}}+g_{\tilde{5}, \tilde{4}}+g_{\widetilde{12}, \widetilde{13}}+g_{\widetilde{13}, \widetilde{12}}+g_{\widetilde{14}, \widetilde{15}}+g_{\widetilde{15}, \widetilde{14}}\right) \\
& +4\left[\left(g_{\tilde{8}, \tilde{10}}+g_{\tilde{10}, \tilde{8}}\right) \sin ^{2} \phi+\left(g_{\tilde{9}, \widetilde{10}}+g_{\widetilde{10}, \tilde{9}}\right) \cos ^{2} \phi\right]
\end{aligned}
$$

and it does not diverge when $T \rightarrow 0$ too.

In order to determine magnetic (longitudinal) susceptibility we should find expressions for Green's functions constructed by the operators $\hat{m}_{i}=2 \hat{\sigma}_{i}^{z}=\hat{n}_{i \uparrow}-\hat{n}_{i \downarrow}$. They can be represented by the electronic functions, because of $\left\langle\hat{m}_{i} \hat{m}_{j}\right\rangle=\left\langle\hat{n}_{i \uparrow} \hat{n}_{j \uparrow}\right\rangle+\left\langle\hat{n}_{i \downarrow} \hat{n}_{j \downarrow}\right\rangle-\left\langle\hat{n}_{i \uparrow} \hat{n}_{j \downarrow}\right\rangle-\left\langle\hat{n}_{i \downarrow} \hat{n}_{j \uparrow}\right\rangle$. Thus in the center of the Brillouin zone, the longitudinal susceptibility is as follows

$$
\begin{aligned}
& \chi_{m}=\sum_{i-j}\left\{\left\langle T \hat{m}_{i}(\tau) \hat{m}_{j}(0)\right\rangle-\left\langle\hat{m}_{i}\right\rangle\left\langle\hat{m}_{j}\right\rangle\right\} \\
& \stackrel{\mathrm{FT}}{=} \frac{2}{T} \delta\left(\omega_{n}\right)\left[w _ { \tilde { 1 } } \left(\frac{1}{4} w_{\tilde{2}}+\frac{1}{4} w_{\tilde{3}}+\frac{1}{4} w_{\tilde{4}}+\frac{1}{4} w_{\tilde{5}}+w_{\tilde{6}}+w_{\tilde{7}}\right.\right. \\
& \left.+\frac{1}{2} w_{\widetilde{12}}+\frac{1}{2} w_{\widetilde{13}}+\frac{1}{2} w_{\widetilde{14}}+\frac{1}{2} w_{\widetilde{15}}\right)+\left(w_{\tilde{2}}+w_{\tilde{3}}\right)\left(\frac{1}{2} w_{\tilde{4}}+\frac{1}{2} w_{\tilde{5}}\right. \\
& \left.+\frac{1}{4} w_{\tilde{8}}+\frac{1}{4} w_{\tilde{9}}+\frac{1}{4} w_{\widetilde{10}}+\frac{1}{4} w_{\widetilde{11}}+w_{\widetilde{14}}+w_{\widetilde{15}}+\frac{1}{4} w_{\widetilde{16}}\right) \\
& +\left(w_{\tilde{4}}+w_{\tilde{5}}\right)\left(\frac{1}{2} w_{\tilde{2}}+\frac{1}{2} w_{\tilde{3}}+\frac{1}{4} w_{\tilde{8}}+\frac{1}{4} w_{\tilde{9}}+\frac{1}{4} w_{\widetilde{10}}+\frac{1}{4} w_{\widetilde{11}}\right. \\
& \left.+w_{\widetilde{12}}+w_{\widetilde{13}}+\frac{1}{4} w_{\widetilde{16}}\right)+w_{\tilde{6}}\left(\frac{1}{4} w_{\tilde{2}}+\frac{1}{4} w_{\tilde{3}}+\frac{9}{4} w_{\tilde{4}}+\frac{9}{4} w_{\tilde{5}}\right. \\
& +2 w_{\tilde{7}}+w_{\tilde{8}}+w_{\tilde{9}}+w_{\widetilde{10}}+w_{\widetilde{11}}+\frac{1}{4} w_{\tilde{12}}+\frac{1}{4} w_{\widetilde{13}}+\frac{9}{4} w_{\widetilde{14}} \\
& \left.+\frac{9}{4} w_{\widetilde{15}}+w_{\widetilde{16}}\right)+w_{\tilde{7}}\left(\frac{9}{4} w_{\tilde{2}}+\frac{9}{4} w_{\tilde{3}}+\frac{1}{4} w_{\tilde{4}}+\frac{1}{4} w_{\tilde{5}}+2 w_{\tilde{6}}\right. \\
& +w_{\tilde{8}}+w_{\tilde{9}}+w_{\widetilde{10}}+w_{\widetilde{11}}+\frac{9}{4} w_{\widetilde{12}}+\frac{9}{4} w_{\widetilde{13}}+\frac{1}{4} w_{\widetilde{14}}+\frac{1}{4} w_{\widetilde{15}} \\
& \left.\left.+\frac{1}{4} w_{\widetilde{10}}+\frac{1}{4} w_{\widetilde{11}}+\frac{1}{2} w_{\widetilde{12}}+\frac{1}{2} w_{\widetilde{13}}+\frac{1}{4} w_{\widetilde{16}}\right)\right] . \\
& \left.+\frac{1}{2} w_{\widetilde{14}}+\frac{1}{2} w_{\widetilde{15}}+\frac{1}{4} w_{\widetilde{16}}\right)+\left(w_{\widetilde{14}}+w_{\widetilde{15}}\right)\left(+\frac{1}{4} w_{\tilde{8}}+\frac{1}{4} w_{\tilde{9}}\right. \\
& \left.+w_{\widetilde{13}}\right)\left(+\frac{1}{4} w_{\tilde{8}}+\frac{1}{4} w_{\tilde{9}}+\frac{1}{4} w_{\widetilde{10}}+\frac{1}{4} w_{\widetilde{11}}\right.
\end{aligned}
$$

The analysis of the expression shows that for concentration $n=1:\left.\chi_{m}\right|_{T \rightarrow 0} \rightarrow \infty$ and the ground state is a symmetric one:

$$
\begin{aligned}
& |\tilde{3}\rangle=\frac{1}{\sqrt{2}}(|3\rangle-|2\rangle)=\frac{1}{\sqrt{2}}(\oint-\Phi), \\
& |\tilde{5}\rangle=\frac{1}{\sqrt{2}}(|5\rangle-|4\rangle)=\frac{1}{\sqrt{2}}(\oint-\oint) .
\end{aligned}
$$

When $n=2:\left.\chi_{m}\right|_{T \rightarrow 0} \rightarrow 0$ and the ground state is a superposition of the $\overrightarrow{R V B}$ and doublon states:

$$
\begin{aligned}
& |\tilde{9}\rangle=\frac{1}{\sqrt{2}}\{\cos \phi(|9\rangle+|10\rangle)-\sin \phi(|8\rangle+|11\rangle)\} \quad(3.31) \\
& =\frac{1}{\sqrt{2}}\{\cos \phi(\Phi+\phi)-\sin \phi(\Phi+\Phi)\}
\end{aligned}
$$

In case of three electrons, as for one, the magnetic susceptibility diverges and the ground state is doubly degenerated (it is a symmetric hole state):

$$
\begin{aligned}
& \widetilde{13}\rangle=\frac{1}{\sqrt{2}}(|13\rangle-|12\rangle)=\frac{1}{\sqrt{2}}(\Phi+\Phi) \\
& |\widetilde{15}\rangle=\frac{1}{\sqrt{2}}(|15\rangle-|14\rangle)=\frac{1}{\sqrt{2}}(\Phi+\Phi)
\end{aligned}
$$

At the edges of the Brillouin zone $\left(q= \pm \frac{\pi}{a}\right)$ susceptibility contains dynamic components only and does not diverge in the limit $T \rightarrow 0$.

$$
\begin{aligned}
\chi_{m} & =\sum_{i-j} e^{\mp i \pi(i-j)}\left\{\left\langle T \hat{m}_{i}(\tau) \hat{m}_{j}(0)\right\rangle-\left\langle\hat{m}_{i}\right\rangle\left\langle\hat{m}_{j}\right\rangle\right\} \\
& \stackrel{\mathrm{FT}}{=}\left(g_{\tilde{2}, \tilde{3}}+g_{\tilde{3}, \tilde{2}}+g_{\tilde{4}, \tilde{5}}+g_{\tilde{5}, \tilde{4}}+g_{\widetilde{12}, \widetilde{13}}\right. \\
& \left.+g_{\widetilde{13}, \widetilde{12}}+g_{\widetilde{14}, \widetilde{15}}+g_{\widetilde{15}, \widetilde{14}}\right) \\
& +2\left[\left(g_{\tilde{8}, \widetilde{10}}+g_{\widetilde{10}, \tilde{8}}+g_{\tilde{9}, \widetilde{11}}+g_{\widetilde{11}, \widetilde{9}}\right) \sin ^{2} \phi\right. \\
& \left.+\left(g_{\tilde{9}, \widetilde{10}}+g_{\widetilde{10}, \tilde{9}}+g_{\tilde{8}, \widetilde{11}}+g_{\widetilde{11}, \tilde{8}}\right) \cos ^{2} \phi\right]
\end{aligned}
$$

The spin-flip operators represented by means of the Hubbard operators on a diagonalized basis are as follows:

$$
\begin{aligned}
\hat{S}_{1}^{+} & =\frac{1}{2} X^{\tilde{4}, \tilde{2}}-\frac{1}{2} X^{\tilde{4}, \tilde{3}}-\frac{1}{2} X^{\tilde{5}, \tilde{2}}+\frac{1}{2} X^{\tilde{5}, \tilde{3}} \\
& -\frac{1}{2} X^{\widetilde{14}, \widetilde{12}}-\frac{1}{2} X^{\widetilde{14}, \widetilde{13}}-\frac{1}{2} X^{\widetilde{15}, \widetilde{12}}+\frac{1}{2} X^{\widetilde{15}, \widetilde{13}} \\
& +\frac{1}{\sqrt{2}} \sin \phi X^{\tilde{7}, \tilde{8}}+\frac{1}{\sqrt{2}} \cos \phi X^{\tilde{7}, \tilde{9}}
\end{aligned}
$$




$$
\begin{aligned}
& -\frac{1}{\sqrt{2}} \sin \phi X^{\tilde{8}, \tilde{6}}-\frac{1}{\sqrt{2}} \cos \phi X^{\tilde{9}, \tilde{6}} \\
& +\frac{1}{\sqrt{2}} X^{\tilde{7}, \widetilde{10}}+\frac{1}{\sqrt{2}} X^{\widetilde{10}, \tilde{6}} \\
\hat{S}_{2}^{+} & =\frac{1}{2} X^{\tilde{4}, \tilde{2}}+\frac{1}{2} X^{\tilde{4}, \tilde{3}}+\frac{1}{2} X^{\tilde{5}, \tilde{2}}+\frac{1}{2} X^{\tilde{5}, \tilde{3}} \\
& -\frac{1}{2} X^{\widetilde{14}, \widetilde{12}}+\frac{1}{2} X^{\widetilde{14}, \widetilde{13}}+\frac{1}{2} X^{\widetilde{15}, \widetilde{12}}-\frac{1}{2} X^{\widetilde{15}, \widetilde{13}} \\
& -\frac{1}{\sqrt{2}} \sin \phi X^{\tilde{7}, \tilde{8}}-\frac{1}{\sqrt{2}} \cos \phi X^{\tilde{7}, \tilde{9}} \\
& +\frac{1}{\sqrt{2}} \sin \phi X^{\tilde{8}, \tilde{6}}+\frac{1}{\sqrt{2}} \cos \phi X^{\tilde{9}, \tilde{6}} \\
& +\frac{1}{\sqrt{2}} X^{\tilde{7}, \widetilde{10}}+\frac{1}{\sqrt{2}} X^{\widetilde{10}, \tilde{6}}
\end{aligned}
$$

and the operator $\hat{S}_{i}^{-}$is a Hermitian conjugate to $\hat{S}_{i}^{+}$.

Similarly, transversal susceptibility is determined in the center of the Brillouin zone by static contributions:

$$
\begin{aligned}
\chi_{s} & =\sum_{i-j}\left\langle T \hat{S}_{i}^{+}(\tau) \hat{S}_{j}^{-}(0)\right\rangle \\
& \stackrel{\mathrm{FT}}{=} \frac{1}{4 T}\left[w_{\tilde{2}}+w_{\tilde{3}}+2 w_{\tilde{6}}+2 w_{\widetilde{10}}+w_{\widetilde{12}}+w_{\widetilde{13}}\right] \delta\left(\omega_{n}\right),
\end{aligned}
$$

that, like a longitudinal susceptibility, diverges when electron concentration is $n=1$ and $n=3$.

At the edge of the Brillouin zone, transversal susceptibility contains dynamic contributions and in the limit $T \rightarrow 0$ it approaches a certain constant value that one can see from the expression:

$$
\begin{aligned}
\chi_{s} & =\left\langle T \hat{S}_{1}^{+}(\tau) \hat{S}_{1}^{-}(0)\right\rangle-\left\langle T \hat{S}_{1}^{+}(\tau) \hat{S}_{2}^{-}(0)\right\rangle \\
& \quad \mathrm{FT} \\
& =g_{\tilde{4}, \tilde{3}}+g_{\tilde{5}, \tilde{2}}+g_{\widetilde{14}, \widetilde{13}}+g_{\widetilde{15}, \widetilde{12}} \\
& +2 \cos ^{2} \phi\left(g_{\tilde{7}, \tilde{9}}+g_{\tilde{9}, \tilde{6}}\right)+2 \sin ^{2} \phi\left(g_{\tilde{7}, \tilde{8}}+g_{\tilde{8}, \tilde{6}}\right) .
\end{aligned}
$$

To conclude, we have calculated electronic, magnetic and spin susceptibilities and investigated their behavior in the limit $T \rightarrow 0$. In the center of the Brillouin zone, the magnetic and spin susceptibilities diverge when electron concentration is $n=1$ and $n=3$, while the charge susceptibility approaches zero at any electron concentrations. At the edge of the Brillouin zone, all susceptibilities approach a constant value for any filling of the cluster.

\section{THE HUBBARD MODEL ON A THREE-SITE CLUSTER}

It is seen from the consideration of a two-site cluster that ferromagnetism can appear in the case of an odd number of electrons in the system. Such a result is in good agreement with Nagaoke solution [22] which has shown that if in the system described by the Hubbard model the number of electrons is $n_{e}=n \pm 1$ ( $n$ is the number of sites) and $U \rightarrow \infty$, the ground state will be ferromagnetic for simple lattices.
Now, let us investigate the Hubbard model on a threesite cluster. In this case, the frustration phenomenon can appear and it is interesting to study how it affects the result.

\section{A. Basic set of equations}

We start from the Hubbard Hamiltonian for a threesite cluster:

$\mathcal{H}=\sum_{i}\left(U \hat{n}_{i \uparrow} \hat{n}_{i \downarrow}-\mu \sum_{\sigma} \hat{n}_{i \sigma}\right)+t \sum_{i \neq j} \sum_{\sigma}\left(\hat{a}_{i \sigma}^{\dagger} \hat{a}_{j \sigma}+\hat{a}_{j \sigma}^{\dagger} \hat{a}_{i \sigma}\right)$

where $\sigma=\uparrow, \downarrow$ and $[i, j]=(1,2,3)$.

The basis of states consists of 64 states which are grouped into sets of states with the same number of electrons. In matrix representation, the Hamiltonian has a block structure. As in the previous case, the basis of states is created by functions in a particle number representation $|p\rangle=\left|n_{1 \uparrow}, n_{1 \downarrow}, n_{2 \uparrow}, n_{2 \downarrow}, n_{3 \uparrow}, n_{3 \downarrow}\right\rangle$ : we have one empty state

$$
|1\rangle=|0,0,0,0,0,0\rangle, \quad \mathcal{H}|1\rangle=0,
$$

six states with one electron

$$
\begin{aligned}
& |2\rangle=|1,0,0,0,0,0\rangle=\hat{a}_{1 \uparrow}^{\dagger}|1\rangle, \\
& |3\rangle=|0,0,1,0,0,0\rangle=\hat{a}_{2 \uparrow}^{\dagger}|1\rangle, \\
& |4\rangle=|0,0,0,0,1,0\rangle=\hat{a}_{3 \uparrow}^{\dagger}|1\rangle, \\
& |5\rangle=|0,1,0,0,0,0\rangle=\hat{a}_{1 \downarrow}^{\dagger}|1\rangle, \\
& |6\rangle=|0,0,0,1,0,0\rangle=\hat{a}_{2 \downarrow}^{\dagger}|1\rangle, \\
& |7\rangle=|0,0,0,0,0,1\rangle=\hat{a}_{3 \downarrow}^{\dagger}|1\rangle, \\
& \mathcal{H}=\left(\begin{array}{cccccc}
-\mu & t & t & 0 & 0 & 0 \\
t & -\mu & t & 0 & 0 & 0 \\
t & t & -\mu & 0 & 0 & 0 \\
0 & 0 & 0 & -\mu & t & t \\
0 & 0 & 0 & t & -\mu & t \\
0 & 0 & 0 & t & t & -\mu
\end{array}\right)
\end{aligned}
$$

nine states with two electrons with opposite spins

$$
\begin{aligned}
& |8\rangle=|1,1,0,0,0,0\rangle=-\hat{a}_{1 \downarrow}^{\dagger}|2\rangle=\hat{a}_{1 \uparrow}^{\dagger}|5\rangle, \\
& \left.|9\rangle=|1,0,0,1,0,0\rangle=-\hat{a}_{2 \downarrow}^{\dagger} \| 2\right\rangle=\hat{a}_{1 \uparrow}^{\dagger}|6\rangle, \\
& |10\rangle=|1,0,0,0,0,1\rangle=-\hat{a}_{3 \downarrow}^{\dagger}|2\rangle=\hat{a}_{1 \uparrow}^{\dagger}|7\rangle, \\
& |11\rangle=|0,1,1,0,0,0\rangle=\hat{a}_{1 \downarrow}^{\dagger}|3\rangle=-\hat{a}_{2 \uparrow}^{\dagger}|5\rangle, \\
& |12\rangle=|0,1,0,0,1,0\rangle=\hat{a}_{1 \downarrow}^{\dagger}|4\rangle=-\hat{a}_{3 \uparrow}^{\dagger}|5\rangle, \\
& |13\rangle=|0,0,1,1,0,0\rangle=-\hat{a}_{2 \downarrow}^{\dagger}|3\rangle=\hat{a}_{2 \uparrow}^{\dagger}|6\rangle, \\
& |14\rangle=|0,0,1,0,0,1\rangle=-\hat{a}_{3 \downarrow}^{\dagger}|3\rangle=\hat{a}_{2 \uparrow}^{\dagger}|7\rangle, \\
& |15\rangle=|0,0,0,1,1,0\rangle=\hat{a}_{2 \downarrow}^{\dagger}|4\rangle=-\hat{a}_{3 \uparrow}^{\dagger}|6\rangle, \\
& |16\rangle=|0,0,0,0,1,1\rangle=-\hat{a}_{3 \downarrow}^{\dagger}|4\rangle=\hat{a}_{3 \uparrow}^{\dagger}|7\rangle,
\end{aligned}
$$




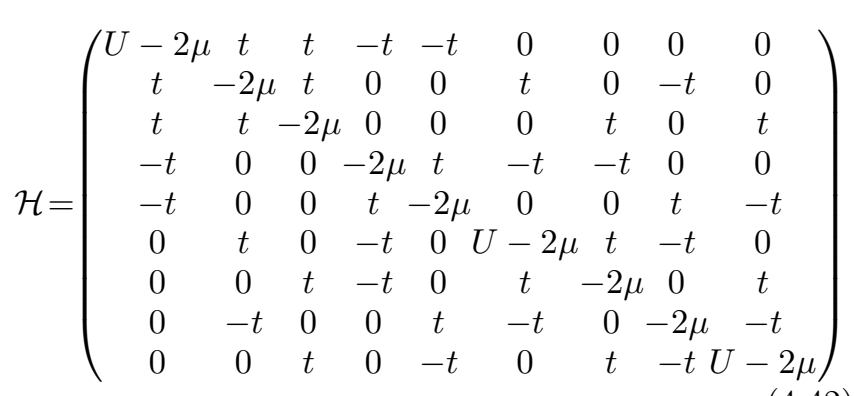

and six states with two electrons with the same spins

$$
\begin{aligned}
& |17\rangle=|1,0,1,0,0,0\rangle=-\hat{a}_{2 \uparrow}^{\dagger}|2\rangle=\hat{a}_{1 \uparrow}^{\dagger}|3\rangle, \\
& |18\rangle=|1,0,0,0,1,0\rangle=-\hat{a}_{3 \uparrow}^{\dagger}|2\rangle=\hat{a}_{1 \uparrow}^{\dagger}|4\rangle, \\
& |19\rangle=|0,0,1,0,1,0\rangle=-\hat{a}_{3 \uparrow}^{\dagger}|3\rangle=\hat{a}_{2 \uparrow}^{\dagger}|4\rangle, \\
& |20\rangle=|0,1,0,1,0,0\rangle=-\hat{a}_{2 \downarrow}^{\dagger}|5\rangle=\hat{a}_{1 \downarrow}^{\dagger}|6\rangle, \\
& |21\rangle=|0,1,0,0,0,1\rangle=-\hat{a}_{3 \downarrow}^{\dagger}|5\rangle=\hat{a}_{1 \downarrow}^{\dagger}|7\rangle, \\
& |22\rangle=|0,1,0,1,0,0\rangle=-\hat{a}_{3 \downarrow}^{\dagger}|6\rangle=\hat{a}_{2 \downarrow}^{\dagger}|7\rangle, \\
& \mathcal{H}=\left(\begin{array}{cccccc}
-2 \mu & t & -t & 0 & 0 & 0 \\
t & -2 \mu & t & 0 & 0 & 0 \\
-t & t & -2 \mu & 0 & 0 & 0 \\
0 & 0 & 0 & -2 \mu & t & -t \\
0 & 0 & 0 & t & -2 \mu & t \\
0 & 0 & 0 & -t & t & -2 \mu
\end{array}\right) .
\end{aligned}
$$

Nine states with three electrons and with the total spin $\frac{1}{2}$

$$
\begin{array}{r}
|23\rangle=|1,1,1,0,0,0\rangle=\hat{a}_{2 \uparrow}^{\dagger}|8\rangle=\hat{a}_{1 \uparrow}^{\dagger}|11\rangle=-\hat{a}_{1 \downarrow}^{\dagger}|17\rangle, \\
|24\rangle=|1,1,0,0,1,0\rangle=\hat{a}_{3 \uparrow}^{\dagger}|8\rangle=\hat{a}_{1 \uparrow}^{\dagger}|12\rangle=-\hat{a}_{1 \downarrow}^{\dagger}|18\rangle, \\
|25\rangle=|1,0,1,1,0,0\rangle=-\hat{a}_{2 \uparrow}^{\dagger}|9\rangle=\hat{a}_{1 \uparrow}^{\dagger}|13\rangle=\hat{a}_{2 \downarrow}^{\dagger}|17\rangle, \\
|26\rangle=|1,0,1,0,0,1\rangle=-\hat{a}_{2 \uparrow}^{\dagger}|10\rangle=\hat{a}_{1 \uparrow}^{\dagger}|14\rangle=\hat{a}_{3 \downarrow}^{\dagger}|17\rangle, \\
|27\rangle=|1,0,0,1,1,0\rangle=\hat{a}_{3 \uparrow}^{\dagger}|9\rangle=\hat{a}_{1 \uparrow}^{\dagger}|15\rangle=-\hat{a}_{2 \downarrow}^{\dagger}|18\rangle, \\
|28\rangle=|1,0,0,0,1,1\rangle=-\hat{a}_{3 \uparrow}^{\dagger}|10\rangle=\hat{a}_{1 \uparrow}^{\dagger}|16\rangle=\hat{a}_{3 \downarrow}^{\dagger}|18\rangle, \\
|29\rangle=|0,1,1,0,1,0\rangle=\hat{a}_{3 \uparrow}^{\dagger}|11\rangle=-\hat{a}_{2 \uparrow}^{\dagger}|12\rangle=\hat{a}_{1 \downarrow}^{\dagger}|19\rangle, \\
|30\rangle=|0,0,1,1,1,0\rangle=\hat{a}_{3 \uparrow}^{\dagger}|13\rangle=\hat{a}_{2 \uparrow}^{\dagger}|15\rangle=-\hat{a}_{2 \downarrow}^{\dagger}|19\rangle, \\
|31\rangle=|0,0,1,0,1,1\rangle=-\hat{a}_{3 \uparrow}^{\dagger}|14\rangle=\hat{a}_{2 \uparrow}^{\dagger}|16\rangle=\hat{a}_{3 \downarrow}^{\dagger}|19\rangle,
\end{array}
$$$$
\begin{aligned}
& \mathcal{H}= \\
& \left(\begin{array}{ccccccccc}
U-3 \mu & t & -t & -t & 0 & 0 & t & 0 & 0 \\
t & U-3 \mu & 0 & 0 & t & -t & -t & 0 & 0 \\
-t & 0 & U-3 \mu & t & -t & 0 & 0 & t & 0 \\
-t & 0 & t & -3 \mu & 0 & t & 0 & 0 & -t \\
0 & t & -t & 0 & -3 \mu & -t & 0 & t & 0 \\
0 & -t & 0 & t & -t & U-3 \mu & 0 & 0 & t \\
t & -t & 0 & 0 & 0 & 0 & -3 \mu & -t & t \\
0 & 0 & t & 0 & t & 0 & -t & U-3 \mu & -t \\
0 & 0 & 0 & -t & 0 & t & t & -t & U-3 \mu
\end{array}\right),
\end{aligned}
$$

one state with three electrons and the total spin $\frac{3}{2}$

$$
\begin{gathered}
|32\rangle=|1,0,1,0,1,0\rangle=\hat{a}_{3 \uparrow}^{\dagger}|17\rangle=\hat{a}_{2 \uparrow}^{\dagger}|18\rangle=\hat{a}_{1 \uparrow}^{\dagger}|19\rangle, \\
\mathcal{H}|32\rangle=-3 \mu|32\rangle .
\end{gathered}
$$

Similarly, for the states with the total spin $-\frac{1}{2}$

$$
\begin{aligned}
& |33\rangle=|1,1,0,1,0,0\rangle=\hat{a}_{2 \downarrow}^{\dagger}|8\rangle=-\hat{a}_{1 \downarrow}^{\dagger}|9\rangle=\hat{a}_{1 \uparrow}^{\dagger}|20\rangle, \\
& |34\rangle=|1,1,0,0,0,1\rangle=\hat{a}_{3 \downarrow}^{\dagger}|8\rangle=-\hat{a}_{1 \downarrow}^{\dagger}|10\rangle=\hat{a}_{1 \uparrow}^{\dagger}|21\rangle, \\
& |35\rangle=|1,0,0,1,0,1\rangle=\hat{a}_{3 \downarrow}^{\dagger}|9\rangle=-\hat{a}_{2 \downarrow}^{\dagger}|10\rangle=\hat{a}_{1 \uparrow}^{\dagger}|22\rangle, \\
& |36\rangle=|0,1,1,1,0,0\rangle=\hat{a}_{2 \downarrow}^{\dagger}|11\rangle=\hat{a}_{1 \downarrow}^{\dagger}|13\rangle=-\hat{a}_{2 \uparrow}^{\dagger}|20\rangle, \\
& |37\rangle=|0,1,1,0,0,1\rangle=\hat{a}_{3 \downarrow}^{\dagger}|11\rangle=\hat{a}_{1 \downarrow}^{\dagger}|14\rangle=-\hat{a}_{2 \uparrow}^{\dagger}|21\rangle, \\
& |38\rangle=|0,1,0,1,1,0\rangle=-\hat{a}_{2 \downarrow}^{\dagger}|12\rangle=\hat{a}_{1 \downarrow}^{\dagger}|15\rangle=\hat{a}_{3 \uparrow}^{\dagger}|20\rangle, \\
& |39\rangle=|0,1,0,0,1,1\rangle=\hat{a}_{3 \downarrow}^{\dagger}|12\rangle=\hat{a}_{1 \downarrow}^{\dagger}|16\rangle=-\hat{a}_{3 \uparrow}^{\dagger}|21\rangle, \\
& |40\rangle=|0,0,1,1,0,1\rangle=\hat{a}_{3 \downarrow}^{\dagger}|13\rangle=-\hat{a}_{2 \downarrow}^{\dagger}|14\rangle=\hat{a}_{2 \uparrow}^{\dagger}|22\rangle, \\
& |41\rangle=|0,0,0,1,1,1,\rangle=\hat{a}_{3 \downarrow}^{\dagger}|15\rangle=\hat{a}_{2 \downarrow}^{\dagger}|16\rangle=-\hat{a}_{3 \uparrow}^{\dagger}|22\rangle,
\end{aligned}
$$

$\mathcal{H}=$

$$
\left(\begin{array}{ccccccccc}
U-3 \mu & t & -t & -t & 0 & t & 0 & 0 & 0 \\
t & U-3 \mu & t & 0 & -t & 0 & -t & 0 & 0 \\
-t & t & U-3 \mu & 0 & 0 & 0 & 0 & t & -t \\
-t & 0 & 0 & -3 \mu & t & -t & 0 & t & 0 \\
0 & -t & 0 & t & -3 \mu & 0 & t & -t & 0 \\
t & 0 & 0 & -t & 0 & U-3 \mu & -t & 0 & t \\
0 & -t & 0 & 0 & t & -t & -3 \mu & 0 & t \\
0 & 0 & t & t & -t & 0 & 0 & U-3 \mu & -t \\
0 & 0 & -t & 0 & 0 & t & t & -t & U-3 \mu
\end{array}\right)
$$

and $-\frac{3}{2}$

$$
\begin{gathered}
|42\rangle=|0,1,0,1,0,1\rangle=\hat{a}_{3 \downarrow}^{\dagger}|20\rangle=-\hat{a}_{2 \downarrow}^{\dagger}|21\rangle=\hat{a}_{1 \downarrow}^{\dagger}|22\rangle, \\
\mathcal{H}|42\rangle=-3 \mu|42\rangle .
\end{gathered}
$$

Nine states with four electrons and with the total spin 0

$$
\begin{aligned}
|43\rangle=|1,1,1,1,0,0\rangle & =-\hat{a}_{2 \downarrow}^{\dagger}|23\rangle=-\hat{a}_{1 \downarrow}^{\dagger}|25\rangle \\
& =\hat{a}_{2 \uparrow}^{\dagger}|33\rangle=\hat{a}_{1 \uparrow}^{\dagger}|36\rangle, \\
|44\rangle=|1,1,1,0,0,1\rangle & =-\hat{a}_{3 \downarrow}^{\dagger}|23\rangle=-\hat{a}_{1 \downarrow}^{\dagger}|26\rangle \\
& =\hat{a}_{2 \uparrow}^{\dagger}|34\rangle=\hat{a}_{1 \uparrow}^{\dagger}|37\rangle, \\
|45\rangle=|1,1,0,1,1,0\rangle & =\hat{a}_{2 \downarrow}^{\dagger}|24\rangle=-\hat{a}_{1 \downarrow}^{\dagger}|27\rangle \\
& =-\hat{a}_{3 \uparrow}^{\dagger}|33\rangle=\hat{a}_{1 \uparrow}^{\dagger}|38\rangle, \\
|46\rangle=|1,1,0,0,1,1\rangle & =-\hat{a}_{3 \downarrow}^{\dagger}|24\rangle=-\hat{a}_{1 \downarrow}^{\dagger}|28\rangle \\
& =\hat{a}_{3 \uparrow}^{\dagger}|34\rangle=\hat{a}_{1 \uparrow}^{\dagger}|39\rangle, \\
|47\rangle=|1,0,1,1,0,1\rangle & =-\hat{a}_{3 \downarrow}^{\dagger}|25\rangle=\hat{a}_{2 \downarrow}^{\dagger}|26\rangle \\
& =-\hat{a}_{2 \uparrow}^{\dagger}|35\rangle=\hat{a}_{1 \uparrow}^{\dagger}|40\rangle,
\end{aligned}
$$




$$
\begin{aligned}
&|48\rangle=|1,0,0,1,1,1\rangle=-\hat{a}_{3 \downarrow}^{\dagger}|27\rangle=-\hat{a}_{2 \downarrow}^{\dagger}|28\rangle \\
&=\hat{a}_{3 \uparrow}^{\dagger}|35\rangle=\hat{a}_{1 \uparrow}^{\dagger}|41\rangle, \\
&|49\rangle=|0,1,1,1,1,0\rangle=\hat{a}_{2 \downarrow}^{\dagger}|29\rangle=\hat{a}_{1 \downarrow}^{\dagger}|30\rangle \\
&=-\hat{a}_{3 \uparrow}^{\dagger}|36\rangle=-\hat{a}_{2 \uparrow}^{\dagger}|38\rangle, \\
&|50\rangle=|0,1,1,0,1,1\rangle=-\hat{a}_{3 \downarrow}^{\dagger}|29\rangle=\hat{a}_{1 \downarrow}^{\dagger}|31\rangle \\
&=\hat{a}_{3 \uparrow}^{\dagger}|37\rangle=-\hat{a}_{2 \uparrow}^{\dagger}|39\rangle, \\
&|51\rangle=|0,0,1,1,1,1\rangle=-\hat{a}_{3 \downarrow}^{\dagger}|30\rangle=-\hat{a}_{2 \downarrow}^{\dagger}|31\rangle \\
& \mathcal{H}=\left(\begin{array}{rrrrrrrrr}
2 U & t & -t & 0 & t & 0 & -t & 0 & 0 \\
t & U & 0 & t & -t & 0 & 0 & t & 0 \\
-t & 0 & U & -t & 0 & t & -t & 0 & 0 \\
0 & t & -t & 2 U & 0 & t & 0 & -t & 0 \\
t & -t & 0 & 0 & U & -t & 0 & 0 & t \\
0 & 0 & t & t & -t & U & 0 & 0 & t \\
-t & 0 & -t & 0 & 0 & 0 & U & -t & -t \\
0 & t & 0 & -t & 0 & 0 & -t & U & -t \\
0 & 0 & 0 & 0 & t & t & -t & -t & 2 U
\end{array}\right)-4 \mu \hat{I} \\
&
\end{aligned}
$$

and six states with the total spin \pm 1

$$
\begin{aligned}
& |52\rangle=|1,1,1,0,1,0\rangle=-\hat{a}_{3 \uparrow}^{\dagger}|23\rangle=\hat{a}_{2 \uparrow}^{\dagger}|24\rangle \\
& =\hat{a}_{1 \uparrow}^{\dagger}|29\rangle=-\hat{a}_{1 \downarrow}^{\dagger}|32\rangle, \\
& |53\rangle=|1,0,1,1,1,0\rangle=-\hat{a}_{3 \uparrow}^{\dagger}|25\rangle=-\hat{a}_{2 \uparrow}^{\dagger}|27\rangle \\
& =\hat{a}_{1 \uparrow}^{\dagger}|30\rangle=\hat{a}_{1 \downarrow}^{\dagger}|32\rangle, \\
& |54\rangle=|1,0,1,0,1,1\rangle=\hat{a}_{3 \uparrow}^{\dagger}|26\rangle=-\hat{a}_{2 \uparrow}^{\dagger}|28\rangle \\
& =\hat{a}_{1 \uparrow}^{\dagger}|31\rangle=-\hat{a}_{3 \downarrow}^{\dagger}|32\rangle, \\
& |55\rangle=|1,1,0,1,0,1\rangle=-\hat{a}_{3 \downarrow}^{\dagger}|33\rangle=\hat{a}_{2 \downarrow}^{\dagger}|34\rangle \\
& =-\hat{a}_{1 \downarrow}^{\dagger}|35\rangle=\hat{a}_{1 \uparrow}^{\dagger}|42\rangle, \\
& |56\rangle=|0,1,1,1,0,1\rangle=-\hat{a}_{3 \downarrow}^{\dagger}|36\rangle=\hat{a}_{2 \downarrow}^{\dagger}|37\rangle \\
& =\hat{a}_{1 \downarrow}^{\dagger}|40\rangle=-\hat{a}_{2 \uparrow}^{\dagger}|42\rangle, \\
& |57\rangle=|0,1,0,1,1,1\rangle=-\hat{a}_{3 \downarrow}^{\dagger}|38\rangle=-\hat{a}_{2 \downarrow}^{\dagger}|39\rangle \\
& =\hat{a}_{1 \downarrow}^{\dagger}|41\rangle=\hat{a}_{3 \uparrow}^{\dagger}|42\rangle, \\
& \mathcal{H}=\left(\begin{array}{cccccc}
U-4 \mu & -t & t & 0 & 0 & 0 \\
-t & U-4 \mu & -t & 0 & 0 & 0 \\
t & -t & U-4 \mu & 0 & 0 & 0 \\
0 & 0 & 0 & U-4 \mu & -t & t \\
0 & 0 & 0 & -t & U-4 \mu & -t \\
0 & 0 & 0 & t & -t & U-4 \mu
\end{array}\right)
\end{aligned}
$$

Six states with five electrons

$$
\begin{aligned}
|58\rangle & =|1,1,1,1,1,0\rangle=\hat{a}_{3 \uparrow}^{\dagger}|43\rangle=\hat{a}_{2 \uparrow}^{\dagger}|45\rangle \\
& =\hat{a}_{1 \uparrow}^{\dagger}|49\rangle=-\hat{a}_{2 \downarrow}^{\dagger}|52\rangle=-\hat{a}_{1 \downarrow}^{\dagger}|53\rangle, \\
|59\rangle & =|1,1,1,0,1,1\rangle=-\hat{a}_{3 \uparrow}^{\dagger}|44\rangle=\hat{a}_{2 \uparrow}^{\dagger}|46\rangle \\
& =\hat{a}_{1 \uparrow}^{\dagger}|50\rangle=\hat{a}_{3 \downarrow}^{\dagger}|52\rangle=-\hat{a}_{1 \downarrow}^{\dagger}|54\rangle,
\end{aligned}
$$

$$
\begin{aligned}
& |60\rangle=|1,0,1,1,1,1\rangle=-\hat{a}_{3 \uparrow}^{\dagger}|47\rangle=-\hat{a}_{2 \uparrow}^{\dagger}|48\rangle \\
& =\hat{a}_{1 \uparrow}^{\dagger}|51\rangle=\hat{a}_{3 \downarrow}^{\dagger}|53\rangle=\hat{a}_{2 \downarrow}^{\dagger}|54\rangle, \\
& |61\rangle=|1,1,1,1,0,1\rangle=\hat{a}_{3 \downarrow}^{\dagger}|43\rangle=-\hat{a}_{2 \downarrow}^{\dagger}|44\rangle \\
& =-\hat{a}_{1 \downarrow}^{\dagger}|47\rangle=\hat{a}_{2 \uparrow}^{\dagger}|55\rangle=\hat{a}_{1 \uparrow}^{\dagger}|56\rangle, \\
& |62\rangle=|1,1,0,1,1,1\rangle=\hat{a}_{3 \downarrow}^{\dagger}|45\rangle=\hat{a}_{2 \downarrow}^{\dagger}|46\rangle \\
& =-\hat{a}_{1 \downarrow}^{\dagger}|48\rangle=-\hat{a}_{3 \uparrow}^{\dagger}|55\rangle=\hat{a}_{1 \uparrow}^{\dagger}|57\rangle, \\
& |63\rangle=|0,1,1,1,1,1\rangle=\hat{a}_{3 \downarrow}^{\dagger}|49\rangle=\hat{a}_{2 \downarrow}^{\dagger}|50\rangle \\
& =\hat{a}_{1 \downarrow}^{\dagger}|51\rangle=-\hat{a}_{3 \uparrow}^{\dagger}|56\rangle=-\hat{a}_{2 \uparrow}^{\dagger}|57\rangle, \\
& \mathcal{H}=\left(\begin{array}{cccccc}
2 U-5 \mu & -t & -t & 0 & 0 & 0 \\
-t & 2 U-5 \mu & -t & 0 & 0 & 0 \\
-t & -t & 2 U-5 \mu & 0 & 0 & 0 \\
0 & 0 & 0 & 2 U-5 \mu & -t & -t \\
0 & 0 & 0 & -t & 2 U-5 \mu & -t \\
0 & 0 & 0 & -t & -t & 2 U-5 \mu
\end{array}\right)
\end{aligned}
$$

Finally, we have one state with six electrons

$$
\begin{gathered}
|64\rangle=|1,1,1,1,1,1\rangle=-\hat{a}_{3 \downarrow}^{\dagger}|58\rangle=-\hat{a}_{2 \downarrow}^{\dagger}|59\rangle \\
=-\hat{a}_{1 \downarrow}^{\dagger}|60\rangle=\hat{a}_{3 \uparrow}^{\dagger}|61\rangle=\hat{a}_{2 \uparrow}^{\dagger}|62\rangle=\hat{a}_{1 \uparrow}^{\dagger}|63\rangle, \\
\mathcal{H}|64\rangle=(3 U-6 \mu)|64\rangle .
\end{gathered}
$$

\section{B. Diagonalization of the Hamiltonian}

The unitary transformation that diagonalizes the Hamiltonian can be constructed by the eigenvectors of this Hamiltonian. The problem on eigenvectors and eigenvalues is solved numerically. We consider the case $U=10 t(U \gg t)$. The small fields $h_{i} \sim 10^{-5}$ at different sites and the chemical potential for different orientation of spins (of the same degree of smallness) with the condition $\sum_{\sigma} \Delta \mu_{\sigma}=0$ were introduced in the Hamiltonian for avoiding the degeneracy of eigenvalues.

Below all energetic quantities are given in units of $t$. For calculating the susceptibilities we use the same formula (2.12). It should be noticed that matrices of the unitary transformation $V^{p \tilde{p}}$ are calculated numerically and have the dimension $64 \times 64$. In Fig. 1 we present a dependence of the electron concentration on the chemical potential value at $T=0$ and $t>0$.

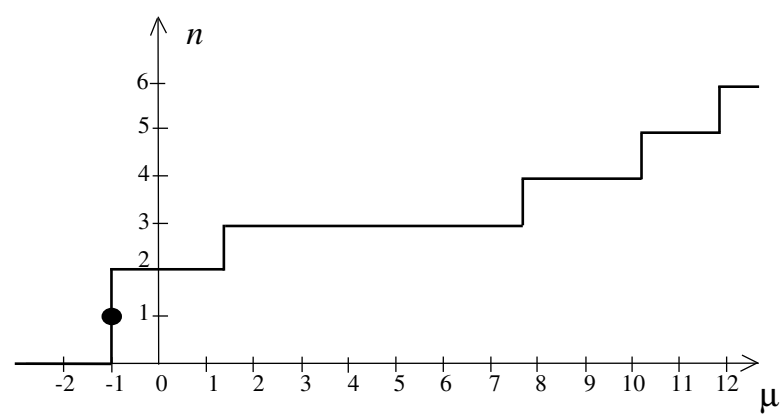

Fig. 1. $n(\mu)$ plot for $t>0$ and $T=0$. 
When $\mu=-1$ we have the degeneracy and states with zero, one and two electrons have the lowest energy simultaneously, but if $\mu$ goes down then the state with " 0 " electrons is the lowest one and if $\mu$ goes up, the state with two electrons will be the ground state. In the case of the negative value of $t$, this dependence is symmetric with regards to the replacements of $\mu \rightarrow(U / 2-\mu)$ and $n \rightarrow(6-n)$.

\section{Numerical results for susceptibilities}

The particle number operators represented by the Hubbard operators on the initial basis are:

$$
\begin{aligned}
& \hat{n}_{1}=\hat{n}_{1 \uparrow}+\hat{n}_{1 \downarrow}=X^{2,2}+X^{5,5}+2 X^{8,8}+X^{9,9} \\
& +X^{10,10}+X^{11,11}+X^{12,12}+X^{17,17}+X^{18,18} \\
& +X^{20,20}+X^{21,21}+2 X^{23,23}+2 X^{24,24}+X^{25,25} \\
& +X^{26,26}+X^{27,27}+X^{28,28}+X^{29,29}+X^{32,32} \\
& +2 X^{33,33}+2 X^{34,34}+X^{35,35}+X^{36,36}+X^{37,37} \\
& +X^{38,38}+X^{39,39}+X^{42,42}+2 X^{43,43}+2 X^{44,44} \\
& +2 X^{45,45}+2 X^{46,46}+X^{47,47}+X^{48,48}+X^{49,49} \\
& +X^{50,50}+2 X^{52,52}+X^{53,53}+X^{54,54}+2 X^{55,55} \\
& +X^{56,56}+X^{57,57}+2 X^{58,58}+2 X^{59,59}+X^{60,60} \\
& +2 X^{61,61}+2 X^{62,62}+X^{63,63}+2 X^{64,64}, \\
& \hat{n}_{2}=\hat{n}_{2 \uparrow}+\hat{n}_{2 \downarrow}=X^{3,3}+X^{6,6}+X^{9,9}+X^{11,11} \\
& +2 X^{13,13}+X^{14,14}+X^{15,15}+X^{17,17}+X^{19,19} \\
& +X^{20,20}+X^{22,22}+X^{23,23}+2 X^{25,25}+X^{26,26} \\
& +X^{27,27}+X^{29,29}+2 X^{30,30}+X^{31,31}+X^{32,32} \\
& +X^{33,33}+X^{35,35}+2 X^{36,36}+X^{37,37}+X^{38,38} \\
& +2 X^{40,40}+X^{41,41}+X^{42,42}+2 X^{43,43}+X^{44,44} \\
& +X^{45,45}+X^{47,47}+X^{48,48}+2 X^{49,49}+X^{50,50} \\
& +2 X^{51,51}+X^{52,52}+2 X^{53,53}+X^{54,54}+X^{55,55} \\
& +2 X^{56,56}+X^{57,57}+2 X^{58,58}+X^{59,59}+2 X^{60,60} \\
& +2 X^{61,61}+X^{62,62}+2 X^{63,63}+2 X^{64,64} \text {, }
\end{aligned}
$$

$$
\begin{aligned}
\hat{n}_{3} & =\hat{n}_{3 \uparrow}+\hat{n}_{3 \downarrow}=X^{4,4}+X^{7,7}+X^{10,10} \\
& +X^{12,12}+X^{14,14}+X^{15,15}+2 X^{16,16}+X^{18,18} \\
& +X^{19,19}+X^{21,21}+X^{22,22}+X^{24,24}+X^{26,26} \\
& +X^{27,27}+X^{28,28}+X^{29,29}+X^{30,30}+2 X^{31,31} \\
& +X^{32,32}+X^{34,34}+X^{35,35}+X^{37,37}+X^{38,38} \\
& +2 X^{39,39}+X^{40,40}+2 X^{41,41}+X^{42,42}+X^{44,44} \\
& +X^{45,45}+X^{46,46}+X^{47,47}+2 X^{48,48}+X^{49,49} \\
& +2 X^{50,50}+2 X^{51,51}+X^{52,52}+X^{53,53}+2 X^{54,54} \\
& +X^{55,55}+X^{56,56}+2 X^{57,57}+X^{58,58}+2 X^{59,59} \\
& +2 X^{60,60}+X^{61,61}+2 X^{62,62}+2 X^{63,63}+2 X^{64,64},
\end{aligned}
$$

Now, let us find electronic susceptibility in the center and out of the center of the Brillouin zone $\left(q=0, \pm \frac{2 \pi}{3 a}\right)$. By means of numerical calculations we find the unitary transformation, which diagonalizes the Hamiltonian. Because we are interested only in the low temperature behavior of susceptibilities, we extract from the large number of terms in (2.12) only the ones that involve the states with the lowest energy. Then we have obtained that for $q=0$ electronic susceptibility equals:

$$
\begin{aligned}
\chi_{c} & =\sum_{i-j}\left\{\left\langle T \hat{n}_{i}(\tau) \hat{n}_{j}(0)\right\rangle-\left\langle\hat{n}_{i}\right\rangle\left\langle\hat{n}_{j}\right\rangle\right\} \\
& \stackrel{\text { FT }}{=}-0.1\left(g_{\widetilde{28}, \widetilde{30}}+g_{\widetilde{30}, \widetilde{28}}\right)+0.12\left(g_{\widetilde{49}, \widetilde{50}}+g_{\widetilde{50}, \widetilde{49}}\right) \\
& +0.2\left(g_{\widetilde{43}, \widetilde{45}}+g_{\widetilde{45}, \widetilde{43}}\right)+0.1\left(g_{\widetilde{45}, \widetilde{47}}+g_{\widetilde{47}, \widetilde{45}}\right) \\
& +0.1\left(g_{\widetilde{48}, \widetilde{51}}+g_{\widetilde{51}, \widetilde{48}}\right)+0.1\left(g_{\widetilde{44}, \widetilde{45}}+g_{\widetilde{45}, \widetilde{44}}\right) \\
& +0.1\left(g_{\widetilde{47}, \widetilde{49}}+g_{\widetilde{49}, \widetilde{47}}\right)+\frac{1}{T}\left[0.9 w_{\tilde{3}} w_{\tilde{4}}+0.9 w_{\tilde{3}} w_{\tilde{7}}\right. \\
& +0.9 w_{\widetilde{4}} w_{\widetilde{6}}+0.9 w_{\widetilde{6}} w_{\tilde{7}}+0.2 w_{\widetilde{14}} w_{\widetilde{17}}+0.2 w_{\widetilde{14}} w_{\widetilde{20}} \\
& \left.+0.2 w_{\widetilde{17}} w_{\widetilde{20}}-0.2 w_{\widetilde{58}} w_{\widetilde{61}}+\text { n.s. }\right] \delta\left(\omega_{n}\right)
\end{aligned}
$$

(n.s. denotes all other components which do not contribute at $T \rightarrow 0$ ).

As is seen from the expression, susceptibility has both dynamic and static components. Analyzing its behavior in the limit $T \rightarrow 0$, one can see that for the concentration $n=1$, the ground states are $\lambda_{\min }=\lambda_{\tilde{3}}=\lambda_{\tilde{4}}=\lambda_{\tilde{6}}=\lambda_{\tilde{7}}$ and susceptibility diverges at $T \rightarrow 0,\left.\chi_{n}\right|_{T \rightarrow 0} \rightarrow \infty$.

For two electrons: $\lambda_{\min }=\lambda_{\widetilde{14}}=\lambda_{\widetilde{17}}=\lambda_{\widetilde{20}}$, and therefore susceptibility diverges in this case too.

In the case of three electrons, the states with lowest energy are $\lambda_{\min }=\lambda_{\widetilde{23}}=\lambda_{\widetilde{24}}=\lambda_{\widetilde{33}}=\lambda_{\widetilde{34}}$ and as a result susceptibility goes to zero.

When we have four electrons $\lambda_{\min }=\lambda_{\widetilde{44}}$ and at $T \rightarrow 0$, susceptibility approaches a constant value.

For five electrons $\lambda_{\min }=\lambda_{\widetilde{58}}=\lambda_{\widetilde{61}}$, and susceptibility diverges for such a cluster filling.

Out of the center of the Brillouin zone $q= \pm \frac{2 \pi}{3 a}$, the electronic susceptibility equals

$$
\begin{aligned}
& \chi_{c}=\sum_{i-j} e^{\mp i \frac{2 \pi}{3}(i-j)}\left\{\left\langle T \hat{n}_{i}(\tau) \hat{n}_{j}(0)\right\rangle-\left\langle\hat{n}_{i}\right\rangle\left\langle\hat{n}_{j}\right\rangle\right\} \\
& \quad \text { FT } 2\left(g_{\tilde{2}, \tilde{3}}+g_{\tilde{3}, \tilde{2}}\right)+3.7\left(g_{\tilde{8}, \tilde{9}}+g_{\tilde{9}, \tilde{8}}\right) \\
& +0.2\left(g_{\tilde{9}, \widetilde{11}}+g_{\widetilde{11}, \tilde{9}}\right)+0.1\left(g_{\tilde{9}, \widetilde{12}}+g_{\widetilde{12}, \tilde{9}}\right) \\
& +0.2\left(g_{\widetilde{23}, \widetilde{28}}+g_{\widetilde{28}, \widetilde{23}}\right)+0.3\left(g_{\widetilde{23}, \widetilde{31}}+g_{\widetilde{31}, \widetilde{23}}\right) \\
& +0.2\left(g_{\widetilde{26}, \widetilde{28}}+g_{\widetilde{28}, \widetilde{26}}\right)+5.9\left(g_{\widetilde{26}, \widetilde{30}}+g_{\widetilde{30}, \widetilde{26}}\right) \\
& +5.8\left(g_{\widetilde{28}, \widetilde{30}}+g_{\widetilde{30}, \widetilde{28}}\right)+\left(g_{\widetilde{12}, \widetilde{12}}+g_{\widetilde{12}, \widetilde{11}}\right) \\
& +3\left(g_{\widetilde{14}, \widetilde{15}}+g_{\widetilde{15}, \widetilde{14}}\right)+0.2\left(g_{\widetilde{46}, \widetilde{47}}+g_{\widetilde{47}, \widetilde{46}}\right) \\
& +2.7\left(g_{\widetilde{49}, \widetilde{50}}+g_{\widetilde{50}, \widetilde{49}}\right)+0.1\left(g_{\widetilde{44}, \widetilde{50}}+g_{\widetilde{50}, \widetilde{44}}\right) \\
& +0.8\left(g_{\widetilde{44}, \widetilde{47}}+g_{\widetilde{47}, \widetilde{44}}\right)+3.4\left(g_{\widetilde{43}, \widetilde{45}}+g_{\widetilde{45}, \widetilde{43}}\right) \\
& +0.1\left(g_{\widetilde{44}, \widetilde{45}}+g_{\widetilde{45}, \widetilde{44}}\right)+0.1\left(g_{\widetilde{48}, \widetilde{51}}+g_{\widetilde{51}, \widetilde{48}}\right) \\
& +0.1\left(g_{\widetilde{47}, \widetilde{49}}+g_{\widetilde{49}, \widetilde{47}}\right)+2\left(g_{\widetilde{58, \widetilde{59}}}+g_{\widetilde{59}, \widetilde{58}}\right)
\end{aligned}
$$




$$
\begin{aligned}
& +\frac{2}{T}\left[0.5 w_{\tilde{3}}+w_{\tilde{9}}+0.2 w_{\widetilde{12}}+0.7 w_{\widetilde{15}}+1.4 w_{\widetilde{26}}\right. \\
& \left.+1.3 w_{\widetilde{28}}+1.1 w_{\widetilde{45}}+0.1 w_{\widetilde{47}}+0.7 w_{\widetilde{50}}+0.5 w_{\widetilde{59}}\right] \delta\left(\omega_{n}\right)
\end{aligned}
$$

and in this case, susceptibility will diverge if there is one electron in the system. In other cases $(n=2,3,4,5,6)$, susceptibility is a finite quantity.

The longitudinal susceptibility is built on the magnetic moment operators which are expressed through the particle number operators by means of the Hubbard operators on the initial basis. They are as follows:

$$
\begin{aligned}
& \hat{m}_{1}=\hat{n}_{1 \uparrow}-\hat{n}_{1 \downarrow}=X^{2,2}-X^{5,5}+X^{9,9}+X^{10,10} \\
& -X^{11,11}-X^{12,12}+X^{17,17}+X^{18,18}-X^{20,20}-X^{21,21} \\
& +X^{25,25}+X^{26,26}+X^{27,27}+X^{28,28}-X^{29,29}+X^{32,32} \\
& +X^{35,35}-X^{36,36}-X^{37,37}-X^{38,38}-X^{39,39}-X^{42,42} \\
& +X^{47,47}+X^{48,48}-X^{49,49}-X^{50,50}+X^{53,53}+X^{54,54} \\
& -X^{56,56}-X^{57,57}+X^{60,60}-X^{63,63}
\end{aligned}
$$$$
\hat{m}_{2}=\hat{n}_{2 \uparrow}-\hat{n}_{2 \downarrow}=X^{3,3}-X^{6,6}-X^{9,9}+X^{11,11}
$$

$+X^{14,14}-X^{15,15}+X^{17,17}+X^{19,19}-X^{20,20}-X^{22,22}$

$+X^{23,23}+X^{26,26}-X^{27,27}+X^{29,29}+X^{31,31}+X^{32,32}$

$-X^{33,33}-X^{35,35}+X^{37,37}-X^{38,38}-X^{41,41}-X^{42,42}$

$+X^{44,44}-X^{45,45}-X^{48,48}+X^{50,50}+X^{52,52}+X^{54,54}$

$-X^{55,55}-X^{57,57}+X^{59,59}-X^{62,62}$

$\hat{m}_{3}=\hat{n}_{3 \uparrow}-\hat{n}_{3 \downarrow}=X^{4,4}-X^{7,7}-X^{10,10}+X^{12,12}$

$-X^{14,14}+X^{15,15}+X^{18,18}+X^{19,19}-X^{21,21}-X^{22,22}$

$+X^{24,24}-X^{26,26}+X^{27,27}+X^{29,29}+X^{30,30}+X^{32,32}$

$-X^{34,34}-X^{35,35}-X^{37,37}+X^{38,38}-X^{40,40}-X^{42,42}$

$-X^{44,44}+X^{45,45}-X^{47,47}+X^{49,49}+X^{52,52}+X^{53,53}$

$-X^{55,55}-X^{56,56}+X^{58,58}-X^{61,61}$.

It the center of the Brillouin zone $(q=0)$ susceptibility contains the static components only:

$$
\begin{aligned}
& \chi_{m}=\sum_{i-j}\left\{\left\langle T \hat{m}_{i}(\tau) \hat{m}_{j}(0)\right\rangle-\left\langle\hat{m}_{i}\right\rangle\left\langle\hat{m}_{j}\right\rangle\right\} \\
& \stackrel{\mathrm{FT}}{=} \frac{1}{T}\left[4 w_{\tilde{3}} w_{\tilde{7}}+4 w_{\tilde{4}} w_{\tilde{7}}+3 w_{\tilde{3}} w_{\tilde{6}}+3 w_{\tilde{4}} w_{\tilde{6}}+16 w_{\widetilde{17}} w_{\widetilde{20}}\right. \\
& +4 w_{\widetilde{14}} w_{\widetilde{17}}+4 w_{\widetilde{14}} w_{\widetilde{20}}+4 w_{\widetilde{23}} w_{\widetilde{33}}+4 w_{\widetilde{24}} w_{\widetilde{33}} \\
& \left.+3 w_{\widetilde{23}} w_{\widetilde{34}}+3 w_{\tilde{24}} w_{\tilde{34}}+4 w_{\tilde{58}} w_{\tilde{61}}+n . s .\right] \delta\left(\omega_{n}\right)
\end{aligned}
$$

and diverges when electron concentration is $n=2,3,5$.

Out of the center of the Brillouin zone $q= \pm \frac{2 \pi}{3 a}$, the magnetic susceptibility contains both dynamic and static terms

$$
\begin{aligned}
\chi_{m} & =\sum_{i-j} e^{\mp i \frac{2 \pi}{3}(i-j)}\left\{\left\langle T \hat{m}_{i}(\tau) \hat{m}_{j}(0)\right\rangle-\left\langle\hat{m}_{i}\right\rangle\left\langle\hat{m}_{j}\right\rangle\right\} \\
& \stackrel{\mathrm{FT}}{=} 2.02\left(g_{\tilde{2}, \tilde{3}}+g_{\tilde{3}, \tilde{2}}\right)+2.01\left(g_{\widetilde{17}, \widetilde{18}}+g_{\widetilde{18}, \widetilde{17}}\right) \\
& +2.96\left(g_{\widetilde{12}, \widetilde{15}}+g_{\widetilde{15}, \widetilde{12}}\right)+2.73\left(g_{\widetilde{11}, \widetilde{15}}+g_{\widetilde{15}, \widetilde{11}}\right)
\end{aligned}
$$

$$
\begin{aligned}
& +0.28\left(g_{\widetilde{8}, \widetilde{15}}+g_{\widetilde{15}, \widetilde{8}}\right)+2.94\left(g_{\widetilde{12}, \widetilde{14}}+g_{\widetilde{14}, \widetilde{12}}\right) \\
& +0.04\left(g_{\widetilde{9}, \widetilde{14}}+g_{\widetilde{14}, \widetilde{9}}\right)+0.01\left(g_{\widetilde{9}, \widetilde{16}}+g_{\widetilde{16}, \widetilde{9}}\right) \\
& +1.99\left(g_{\widetilde{26}, \widetilde{28}}+g_{\widetilde{28}, \widetilde{26}}\right)+1.92\left(g_{\widetilde{26}, \widetilde{30}}+g_{\widetilde{30}, \widetilde{26}}\right) \\
& +1.92\left(g_{\widetilde{28}, \widetilde{30}}+g_{\widetilde{30}, \widetilde{28}}\right)+7.56\left(g_{\widetilde{23}, \widetilde{25}}+g_{\widetilde{25}, \widetilde{23}}\right) \\
& +0.24\left(g_{\widetilde{25}, \widetilde{28}}+g_{\widetilde{28}, \widetilde{25}}\right)+0.2\left(g_{\widetilde{25}, \widetilde{26}}+g_{\widetilde{26}, \widetilde{25}}\right) \\
& +0.11\left(g_{\widetilde{23}, \widetilde{30}}+g_{\widetilde{30}, \widetilde{23}}\right)+0.08\left(g_{\widetilde{23}, \widetilde{28}}+g_{\widetilde{28}, \widetilde{23}}\right) \\
& +2.01\left(g_{\widetilde{52}, \widetilde{53}}+g_{\widetilde{53}, \widetilde{52}}\right)+2.94\left(g_{\widetilde{47}, \widetilde{50}}+g_{\widetilde{50}, \widetilde{47}}\right) \\
& +2.85\left(g_{\widetilde{44}, \widetilde{50}}+g_{\widetilde{50}, \widetilde{44}}\right)+0.14\left(g_{\widetilde{43}, \widetilde{50}}+g_{\widetilde{50}, \widetilde{43}}\right) \\
& +2.92\left(g_{\widetilde{47}, \widetilde{49}}+g_{\widetilde{49}, \widetilde{47}}\right)+0.08\left(g_{\widetilde{45}, \widetilde{49}}+g_{\widetilde{49}, \widetilde{45}}\right) \\
& +0.06\left(g_{\widetilde{45}, \widetilde{50}}+g_{\widetilde{50}, \widetilde{45}}\right)+2.02\left(g_{\widetilde{58}, \widetilde{59}}+g_{\widetilde{59}, \widetilde{58}}\right) \\
& +\frac{2}{T}\left[0.49 w_{\widetilde{3}}+0.49 w_{\widetilde{18}}+1.84 w_{\widetilde{23}}+0.49 w_{\widetilde{53}}\right. \\
& +0.49 w_{\widetilde{59}} \delta\left(\omega_{n}\right)
\end{aligned}
$$

Hence, for $n=3$ the static part of susceptibility diverges out of the center of the Brillouin zone. At temperatures close to zero, the dynamic part of susceptibility converges to a positive constant for any filling of the cluster.

And at last the spin (transverse) susceptibility is built on the spin-flip operators. On the initial basis:

$$
\begin{aligned}
\hat{S}_{1}^{+} & =\hat{a}_{1 \uparrow}^{\dagger} \hat{a}_{1 \downarrow}=X^{2,5}+X^{9,20}+X^{10,21}+X^{17,11} \\
& +X^{18,12}+X^{25,36}+X^{26,37}+X^{27,38}+X^{28,39} \\
& +X^{32,29}+X^{35,42}+X^{47,56}+X^{48,57}+X^{53,49} \\
& +X^{54,50}+X^{60,63}
\end{aligned}
$$

$$
\begin{aligned}
\hat{S}_{2}^{+} & =\hat{a}_{2 \uparrow}^{\dagger} \hat{a}_{2 \downarrow}=X^{3,6}+X^{11,20}+X^{14,22}+X^{17,9} \\
& +X^{19,15}+X^{23,33}+X^{26,35}+X^{29,38}+X^{31,41} \\
& +X^{32,27}+X^{37,42}+X^{44,55}+X^{50,57}+X^{52,45} \\
& +X^{54,48}+X^{59,62}
\end{aligned}
$$

$$
\begin{aligned}
\hat{S}_{3}^{+} & =\hat{a}_{3 \uparrow}^{\dagger} \hat{a}_{3 \downarrow}=X^{4,7}+X^{12,21}+X^{15,22}+X^{18,10} \\
& +X^{19,14}+X^{24,34}+X^{27,35}+X^{29,37}+X^{30,40} \\
& +X^{32,26}+X^{38,42}+X^{45,55}+X^{49,56}+X^{52,44} \\
& +X^{53,47}+X^{58,61} .
\end{aligned}
$$

If we switch to a new basis and do all the necessary operations, we will obtain that in the center of the Brillouin zone, susceptibility is as follows:

$$
\begin{aligned}
\chi_{s} & =\sum_{i-j}\left\langle T \hat{S}_{i}^{+}(\tau) \hat{S}_{j}^{-}(0)\right\rangle \\
& \stackrel{\mathrm{FT}}{=} \frac{2}{T}\left[w_{\tilde{2}}+w_{\tilde{3}}+w_{\tilde{4}}+4 w_{\widetilde{14}}+4 w_{\widetilde{15}}+4 w_{\widetilde{16}}\right. \\
& +1.024 w_{\widetilde{23}}+1.024 w_{\widetilde{24}}+7 w_{\widetilde{25}}+w_{\widetilde{26}}+w_{\widetilde{27}}+w_{\widetilde{28}} \\
& +w_{\widetilde{29}}+w_{\widetilde{30}}+w_{\widetilde{31}}+3 w_{\widetilde{35}}+4 w_{\widetilde{49}}+4 w_{\widetilde{50}}+4 w_{\widetilde{51}} \\
& \left.+w_{\widetilde{58}}+w_{\widetilde{59}}+w_{\widetilde{60}}\right] \delta\left(\omega_{n}\right)
\end{aligned}
$$


At the temperatures close to zero, it is obtained that for the concentration $n=2 \lambda_{\min }=\lambda_{\widetilde{14}}=\lambda_{\widetilde{17}}=\lambda_{\widetilde{20}}$ and susceptibility tends to infinity. This states can be represented as a superposition of the initial states.

$$
\begin{aligned}
|\widetilde{14}\rangle & =10^{-7}|8\rangle-0.4|9\rangle+0.4|10\rangle-0.4|11\rangle \\
& +0.4|12\rangle+10^{-6}|13\rangle-0.4|14\rangle-0.4|15\rangle-10^{-7}|16\rangle
\end{aligned}
$$

is a superposition of the states with two opposite spins at different sites (something like an itinerant RVB bound) and

$$
\begin{aligned}
& |\widetilde{17}\rangle=0.58|17\rangle-0.58|18\rangle+0.58|19\rangle \\
& =0.58+198 \\
& |\widetilde{20}\rangle=0.58|20\rangle-0.58|21\rangle+0.58|22\rangle
\end{aligned}
$$

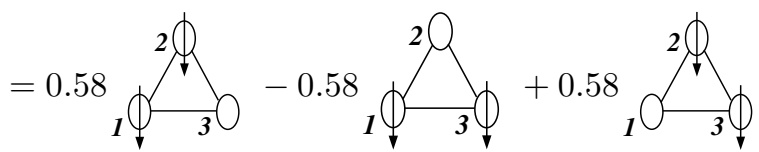

states with parallel spins.

For $n=3,\left.\chi_{m, s}\right|_{T \rightarrow 0} \rightarrow \infty$ and the relative states reflect frustration

$$
\begin{aligned}
|\widetilde{23}\rangle & =-0.1|23\rangle+0.02|24\rangle+0.1|25\rangle-0.8|26\rangle \\
& +0.37|27\rangle+0.12|28\rangle+0.42|29\rangle-0.01|30\rangle-0.13|31\rangle \\
& \\
& +0.7|27\rangle+0.05|28\rangle-0.67|29\rangle-0.13|30\rangle+0.04|31\rangle
\end{aligned}
$$

$$
\begin{aligned}
& |\widetilde{33}\rangle=-0.1|33\rangle+0.04|34\rangle-0.5|35\rangle+0.08|36\rangle \\
& -0.25|37\rangle+0.77|38\rangle+0.1|39\rangle+0.004|40\rangle-0.13|41\rangle
\end{aligned}
$$$$
|\widetilde{34}\rangle=0.06|33\rangle-0.12|34\rangle+0.6|35\rangle+0.1|36\rangle
$$

$$
-0.75|37\rangle+0.15|38\rangle-0.07|39\rangle-0.1|40\rangle+0.02|41\rangle
$$

For the electron concentration $n=4: \lambda_{\min }=\lambda_{\widetilde{43}}$, and the ground state is

$$
\begin{aligned}
\widetilde{43}\rangle & =-0.12|43\rangle+0.4|44\rangle-0.4|45\rangle-0.12|46\rangle \\
& +0.4|47\rangle+0.4|48\rangle-0.4|49\rangle-0.4|50\rangle+0.12|51\rangle
\end{aligned}
$$

When $n=5, \lambda_{\min }=\lambda_{\widetilde{58}}=\lambda_{\widetilde{61}}$ then $\left.\chi_{m, s}\right|_{T \rightarrow 0} \rightarrow \infty$ and the relative states are

$$
\begin{aligned}
& |\widetilde{58}\rangle=0.58|58\rangle+0.58|59\rangle+0.58|60\rangle \\
& =0.58+2 \\
& |\widetilde{61}\rangle=0.58|61\rangle+0.58|62\rangle+0.58|63\rangle
\end{aligned}
$$

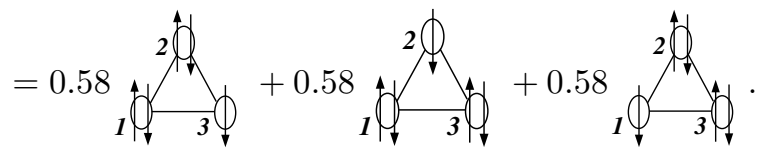

Out of the center of the Brillouin zone $q= \pm \frac{2 \pi}{3 a}$ the susceptibility contains both static and dynamic components.

$$
\begin{aligned}
& \chi_{s}=\sum_{i-j} e^{\mp i \frac{2 \pi}{3}(i-j)}\left\langle T \hat{S}_{i}^{+}(\tau) \hat{S}_{j}^{-}(0)\right\rangle \\
& \quad \text { FT } \frac{1}{T}\left[0.3 w_{\widetilde{3}}+0.3 w_{\widetilde{15}}+1.25 w_{\widetilde{23}}+0.3 w_{\widetilde{51}}+0.3 w_{\widetilde{59}}\right] \delta\left(\omega_{n}\right) \\
& +0.3\left(g_{\widetilde{14}, \widetilde{22}}+g_{\widetilde{22}, \widetilde{14}}\right)+0.5\left(g_{\widetilde{12}, \widetilde{22}}+g_{\widetilde{22}, \widetilde{12}}\right) \\
& +0.5\left(g_{\widetilde{12}, \widetilde{17}}+g_{\widetilde{17}, \widetilde{12}}\right)+0.5\left(g_{\widetilde{11}, \widetilde{22}}+g_{\widetilde{22}, \widetilde{11}}\right) \\
& +\left(g_{\widetilde{24}, \widetilde{32}}+g_{\widetilde{32}, \widetilde{24}}\right)+0.3\left(g_{\widetilde{30}, \widetilde{36}}+g_{\widetilde{36}, \widetilde{30}}\right) \\
& +0.3\left(g_{\widetilde{27}, \widetilde{38}}+g_{\widetilde{38}, \widetilde{27}}\right)+0.3\left(g_{\widetilde{30}, \widetilde{38}}+g_{\widetilde{38}, \widetilde{30}}\right) \\
& +0.3\left(g_{\widetilde{23}, \widetilde{35}}+g_{\widetilde{35}, \widetilde{23}}\right)+0.3\left(g_{\widetilde{51}, \widetilde{55}}+g_{\widetilde{55}, \widetilde{51}}\right) \\
& +0.5\left(g_{\widetilde{44}, \widetilde{56}}+g_{\widetilde{56}, \widetilde{44}}\right)+0.5\left(g_{\widetilde{47}, \widetilde{56}}+g_{\widetilde{56}, \widetilde{47}}\right) \\
& +0.5\left(g_{\widetilde{47}, \widetilde{55}}+g_{\widetilde{55}, \widetilde{47}}\right)+0.3\left(g_{\widetilde{58}, \widetilde{62}}+g_{\widetilde{62}, \widetilde{58}}\right)
\end{aligned}
$$

Static contribution to the transverse susceptibility diverges out of the center of the Brillouin zone only if there are three electrons in the system and dynamical contribution to susceptibility approaches a constant value for any filling of the cluster.

\section{SUMMARY}

The Hubbard model on two- and three-site clusters is investigated. For the narrow conduction band $(U \gg t)$, 
susceptibilities are determined and their behavior is investigated in the case of $T \rightarrow 0$. It is seen from the consideration of a two-site cluster that ferromagnetism can appear in the case of an odd number of electrons in the system and only in the center of the Brillouin zone. Such a result is in good agreement with the Nagaoke solution [22], which has shown that if in the system described by the Hubbard model, the number of electrons is $n_{e}=n \pm 1$ ( $n$ is the number of sites) and $U \rightarrow \infty$, the ground state will be ferromagnetic for simple lattices.
In the case of a three-site cluster in the center of the Brillouin zone susceptibilities diverge both in the cases of odd and even numbers of electrons in the system. Beyond the center of the Brillouin zone a modulated magnetic structure can appear at half-filling as well. Such results show that for a three-site cluster when the ferromagnetic state is destroyed by frustration the Nagaoke solution is not valid any more and such a behavior can be predicted for larger frustrated lattices.
[1] J. Hubbard, Proc. Roy. Soc. A276, 238 (1963).

[2] J. Hubbard, Proc. Roy. Soc. A277, 237 (1964).

[3] J. Hubbard, Proc. Roy. Soc. A281, 401 (1964).

[4] P. W. Anderson, Science 235, 1196 (1987).

[5] P. W. Anderson, Science 256, 1526 (1992).

[6] E. H. Lieb, F. Y. Wu, Phys. Rev. Lett. 20, 1445 (1968).

[7] P. Schlottmann, Int. J. Mod. Phys. B 11, 355 (1997).

[8] A. Georges, G. Kotliar, W. Krauth, M. J. Rozenberg, Rev. Mod. Phys. 68, 13 (1996).

[9] E. Kovács, Z. Gulácsi, Phil. Mag. 86, 2073 (2006).

[10] K. Capelle, L. N. Oliveira, Phys. Rev. B 73, 113111 (2006).

[11] A. Harris, R. Lange, Phys. Rev. 157, 295 (1967).

[12] A. M. Shvaika, Phys. Rev. B 62, 2358 (2000).

[13] A. Avella, F. Mancini, T. Saikawa, Eur. Phys. J. B 36,
445 (2003).

[14] R. Schumann, Ann. Phys. (Leipzig) 11, 49 (2002).

[15] H. Shiba, P. A. Pincus, Phys. Rev. B 5, 1966 (1972).

[16] R. Baxter, Exactly solved models in statistical mechanics (Mir, Moscow, 1985).

[17] M. Capone, L. Capriotti, F. Becca, S. Caprara, Phys. Rev. B 63, 085104 (2001).

[18] S. Kondo et al., Phys. Rev. Lett. 78, 3729 (1997).

[19] R. Ballou, E. Lelièvre-Berna, B. Fåk, Phys. Rev. Lett. 76, 2125 (1996).

[20] Y. Imai, N. Kawakami, Phys. Rev. B 65, 233103 (2002)

[21] L. D. Didukh, L. F. Pryadko, I. V. Stasyuk, Correlation effects in narrow-band materials (Vyshcha shkola, Lviv, 1978).

[22] Y. Nagaoka, Phys. Rev. 147, 392 (1966).

\title{
ЗАРЯДОВІ ТА МАГНЕТНІ СТАНИ МОДЕЛІ ГАББАРДА НА ТРИВУЗЛОВОМУ КЛАСТЕРІ
}

\author{
О. П. Матвєєв, А. М. Швайка \\ Інститут фізики конденсованих систем Національної академї наук Украӥни, \\ вул. Свениічького, 1, Лъвів, 79011, Україна
}

\begin{abstract}
Досліджено модель Габбарда на кластерах малого розміру. Методом функцій Гріна розраховано електронну, магнетну та спінову сприйнятливості й вивчено їхню поведінку при низьких температурах, зокрема температурну поведінку сприйнятливостей для дво- та тривузлового кластерів на краю та в центрі зони Бріллюена. Отримано, що якщо при заданій концентрації основний стан є поляризованим, то сприйнятливості розбігаються за законом Кюрі й система переходить у магнетовпорядкований стан при $T=0$.
\end{abstract}

